\title{
The complete spectrum of the equatorial electrojet related to solar tides: CHAMP observations
}

\author{
H. Lühr ${ }^{1}$ and C. Manoj ${ }^{2}$ \\ ${ }^{1}$ GFZ, German Research Centre for Geosciences, Potsdam, Germany \\ ${ }^{2}$ National Geophysical Data Center, NOAA, Boulder, Colorado, USA \\ Correspondence to: H. Lühr (hluehr@gfz-potsdam.de)
}

Received: 21 February 2013 - Revised: 24 May 2013 - Accepted: 2 July 2013 - Published: 5 August 2013

\begin{abstract}
Based on $10 \mathrm{yr}$ of magnetic field measurements by the CHAMP satellite we draw a detailed picture of the equatorial electrojet (EEJ) tidal variations. For the first time the complete EEJ spectrum related to average solar tides has been compiled. A large fraction of the resulting spectrum is related to the switch on/off of the EEJ between day and night. This effect has carefully been considered when interpreting the results. As expected, largest amplitudes are caused by the migrating tides representing the mean diurnal variation. Higher harmonics of the daily variations show a $1 / f$ falloff in amplitude. Such a spectrum is required to represent the vanishing of the EEJ current at night. The migrating tidal signal exhibits a distinct annual variation with large amplitudes during December solstice and equinox seasons but a depression by a factor of 1.7 around June-July. A rich spectrum of non-migrating tidal effects is deduced. Most prominent is the four-peaked longitudinal pattern around August. Almost $90 \%$ of the structure can be attributed to the diurnal eastward-propagating tide DE3. In addition the westwardpropagating DW5 is contributing to wave- 4 . The secondlargest non-migrating tide is the semi-diurnal SW4 around December solstice. It causes a wave- 2 feature in satellite observations. The three-peaked longitudinal pattern, often quoted as typical for the December season, is significantly weaker. During the months around May-June a prominent wave-1 feature appears. To first order it represents a stationary planetary wave SPW1 which causes an intensification of the EEJ at western longitudes beyond $60^{\circ} \mathrm{W}$ and a weakening over Africa/India. In addition, a prominent terdiurnal non-migrating tide TW4 causes the EEJ to peak later, at hours past 14:00 local time in the western sector. A particularly interesting non-migrating tide is the semi-diurnal SW3. It causes largest EEJ amplitudes from October through
\end{abstract}

December. This tidal component shows a strong dependence on solar flux level with increasing amplitudes towards solar maximum. We are not aware of any previous studies mentioning this behaviour of SW3. The main focus of this study is to present the observed EEJ spectrum and its relation to tidal driving. For several of the identified spectral components we cannot offer convincing explanations for the generation mechanisms.

Keywords. Ionosphere (Ionosphere-atmosphere interactions)

\section{Introduction}

The equatorial electrojet (EEJ) represents a narrow ribbon of intense ionospheric currents above the magnetic dip equator. It is a phenomenon caused primarily by the strongly enhanced (Cowling) conductivity in combination with the large-scale polarisation electric field and the local effects of tidal winds in the ionospheric $\mathrm{E}$ layer. The periodic dynamo action is modulated by the diurnal variation of the ionospheric conductivity. The electrodynamics related to the EEJ has been a topic of research since its early observations (e.g. Cowling, 1933; Sugiura and Poros, 1969; Richmond, 1973; Maus et al., 2007) and is still an issue (e.g. England et al., 2010). The main driver is the upper-atmospheric tidal circulation moving together with the Sun around the Earth. The EEJ responds only during daytime to the wind system because of the vanishing E region conductivity at night. This control of the EEJ by the solar tide has been recognised early on. Soon it was discovered that also the lunar tide influences the intensity of the EEJ (e.g. Bartels and Johnson, 1940, and references therein). In a comprehensive study Lühr et 
al. (2012b) investigated the average characteristics of lunar tidal effects on the EEJ based on $10 \mathrm{yr}$ of CHAMP satellite magnetic field observations. They found that the lunar modulation of the EEJ intensity amounts generally to about $15 \%$ of the amplitude. Exceptions are periods after northern hemispheric sudden stratospheric warming (SSW) events which occur predominately during the months December, January, and February. Park et al. (2012) have shown that the lunar tidal effect on the EEJ can be increased by a factor of 5 during SSW events. The strongly enhanced influence of the moon on the EEJ strength during times following SSWs makes the effect quite obvious and is probably the reason for the significant interest in lunar tidal studies of the electrojet during past decades (e.g. Rastogi and Triveldi, 1970). Furthermore, the lunitidal effect can be observed equally well by any observatory under the EEJ. In this study we will not consider lunar tides.

Only recently signatures of non-migrating solar tides were identified in EEJ measurements. England et al. (2006) were the first who related a wavenumber 4 longitudinal pattern of EEJ intensity, derived from CHAMP magnetic field observations, with the diurnal eastward-propagating tidal component, DE3. By including tidal phase information in the analysis Lühr et al. (2008) confirmed the DE3 origin of these ambiguous satellite observations. They furthermore showed that the DE3 influence is strong during equinoxes but is absent during the months around December solstice. In a follow-up paper Lühr et al. (2012a) included in their tidal study, in addition to the EEJ, the vertical plasma drift and the equatorial ionisation anomaly. They presented the annual variation of several important non-migrating tidal components. DE3 was found to be strongest in all three ionospheric quantities during the months August and September and absent around December solstice. Also westward-propagating non-migrating tides were found exhibiting largest amplitudes predominately during solstice months. They are believed to be of quite different character.

Based on global atmospheric model simulations Hagan and Forbes (2002) predicted the existence of DE3 tidal waves in the upper atmosphere excited by latent heat release in the tropical troposphere. These predictions have later been confirmed by wind observations in the mesosphere, lower thermosphere (MLT) (e.g. Oberheide et al., 2006) and temperature measurements (e.g. Forbes et al., 2008). More recently it has been shown that also longitudinal variations in solar radiative input play a significant role in the generation of these non-migrating tides (Zhang et al., 2010). Unexpectedly, clear DE3 signatures were also detected in low-latitude zonal winds at $400 \mathrm{~km}$ altitude (e.g. Häusler and Lühr, 2009). It is still not fully understood which fraction of the wind signal is propagating directly up from the troposphere and which is transmitted through ion/neutral coupling into the upper thermosphere. From all these observations it can be concluded that tropospheric phenomena can have significant influence on the upper-atmospheric dynamics.
Tidal studies of neutral atmospheric quantities have the advantage that the signal is present during the whole day. Conversely, ionospheric signatures are strongly modulated by the day/night contrast of the E region conductivity; i.e. day and night signals have a totally different character and require an independent interpretation. The tidal spectrum of the neutral quantities can be determined directly from the signal distribution in longitude versus local time space with the help of a 2-D Fourier transform. This approach cannot be applied to the EEJ data because sensible readings are only available for daytime hours. In order to retrieve tidal information from this patchy dataset Lühr et al. (2008, 2012a) fitted functions of pre-defined tidal components to the data. Only a limited number ( 2 to 3 ) of prominent tidal components for each wavenumber could be included in the analysis, in order to avoid ambiguities. Therefore, a complete and unbiased tidal spectrum of the EEJ is still missing.

The EEJ can be regarded as the link between wind and electric field in the equatorial $\mathrm{E}$ layer during daytime. The close similarity between tidal features in zonal wind and in EEJ data has been shown by Lühr et al. (2012a) for the annual variation and by Häusler et al. (2013) for interannual variability. Furthermore, Alken and Maus (2010) have successfully demonstrated how to estimate the equatorial electric field from EEJ measurements when the wind at the E region altitudes is known. Since it is difficult to make direct electric field measurements in the E layer, the EEJ intensity can be regarded as a suitable proxy for deriving the characteristics of this highly relevant quantity.

It is known that the EEJ is not only driven by tidal winds. Additional contributions come for example from the interplanetary electric field via the prompt penetration effect. In a statistical study Manoj et al. (2008) have quantified the typical features of this phenomenon at equatorial latitudes for the Jicamarca longitude. The electric field transfer function peaks at periods of about $2 \mathrm{~h}$. At this period the amplitude is attenuated by $25 \mathrm{~dB}$. This means, a typical interplanetary electric field variation of $\pm 1 \mathrm{mV} \mathrm{m}^{-1}$ appears in the $\mathrm{E}$ region as $\pm 0.056 \mathrm{mV} \mathrm{m}^{-1}$, which is about $10 \%$ of the diurnal variation. Over long data series such a moderate signal averages out. Furthermore, the EEJ can be strongly perturbed (predominately suppressed) during magnetic storms (e.g. Kikuchi et al., 2003, 2008). In order to reduce the disturbing influence of magnetic storms we make use of a robust spectral analysis approach (see Sect. 2).

This paper provides a complete spectrum of the EEJ variations induced by solar tides. Until now such a comprehensive picture has not been presented. This is mainly due to insufficient data availability in the past. As opposed to lunar tides the evaluation of the solar tides requires a dense longitudinal coverage of the EEJ at all seasons and local times. Figure 1 shows the longitudinal distribution of the EEJ strength for the four seasons. The patterns vary strongly over the course of a year. CHAMP with its $10 \mathrm{yr}$ of high-resolution magnetic field measurements provided the opportunity to 

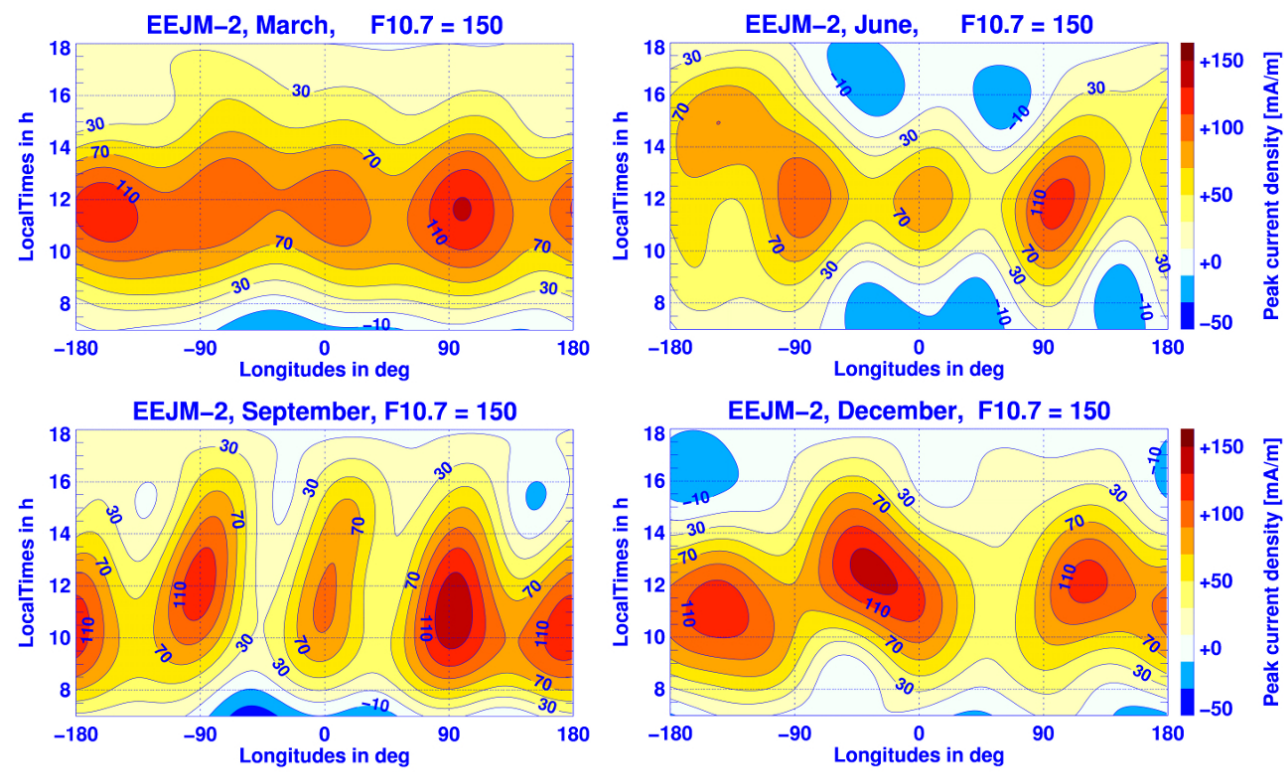

Fig. 1. Longitudinal distribution of the electrojet intensity versus local time. Data for the four seasons are from the EEJM-2 model.

construct an empirical model of the quiet-time EEJ intensity, termed EEJM (Alken and Maus, 2007). Figure 1 is derived from the updated version EEJM-2, which is accessible at http://www.geomag.us/models/EEJ.html. For the studies presented here we consider the multi-year CHAMP EEJ dataset in one go, thus suppressing the day-to-day variability but emphasising the regular cyclic oscillations. In this way we intend to determine reliably the solar tidal signals, which are the prime drivers of the quiet-time EEJ.

In the next section we shortly introduce the CHAMP mission, describe the approach for obtaining the EEJ current density, and outline the analysis for retrieving the tidal spectrum. In Sect. 3 we present average EEJ spectra for high and low solar activity periods. A detailed description of the various spectral components, both their amplitudes and phases, is given in Sect. 4. Then an attempt is made in Sect. 5 to interpret groups of spectral components that seem to belong to the same tidal phenomena. The results obtained are discussed in a wider context in Sect. 6 and compared to previous publications. Main conclusions are summarised in Sect. 7.

\section{The dataset and processing approach}

The CHAMP satellite was launched on 15 July 2000 into a circular, near-polar (inclination $87.3^{\circ}$ ) orbit at $456 \mathrm{~km}$ altitude (Reigber et al., 2002). The orbit slowly decayed until the re-entry of the satellite into the atmosphere on 19 September 2010. Due to the chosen inclination the orbital plane precessed through local time at a rate of $1 \mathrm{~h}$ per 11 days, requiring about 261 days to cover all local times.

To determine the electrojet current density, the magnetic field components are used, as measured by the Fluxgate magnetometer on CHAMP. This instrument samples the three components of the field at $50 \mathrm{~Hz}$ with a resolution of $0.1 \mathrm{nT}$. In order to ensure continuous precision the Fluxgate data are routinely calibrated against the absolute scalar Overhauser readings and then averaged to $1 \mathrm{~Hz}$ samples. A set of two star cameras determined the spacecraft attitude with arcsecond resolution.

CHAMP magnetic field data can be inverted to derive meridional current density profiles of horizontal ionospheric currents (e.g. Ritter et al., 2004; Lühr et al., 2004). This approach takes into account the satellite altitude and various $F$ region plasma effects that affect the magnetic signal (e.g. Lühr et al., 2003). Thus, the decay of the CHAMP orbit and systematic changes of the ionospheric $\mathrm{F}$ region should not influence the obtained current intensities. CHAMP current density profiles showed that the EEJ peaks exactly at the magnetic dip equator at all local times, seasons, and longitudes (Lühr et al., 2004). To characterise the EEJ intensity, we use the peak current density. That means our dataset contains one sample per equator crossing on the dayside occurring every $93 \mathrm{~min}$.

For this study we employed EEJ readings from 59244 equatorial crossings of CHAMP from 1 August 2000 to 1 September 2010. The time series derived from CHAMP represents an asynoptic sampling (measurement taken at a single location at a given time) of the EEJ. It then follows that the spatial and the temporal signals are mixed together in the EEJ data. If one treats the CHAMP data as a pure time series and converts it, e.g. by Fourier transform, into the frequency domain, the stationary wave components in the E region will appear at integer frequencies corresponding to their wavenumber. Migrating wave components on the other 
hand appear Doppler-shifted to lower (higher) frequencies for westward (eastward)-moving components (e.g. Salby, 1982) in frequency spectra. For example, Park at al. (2012) showed that the lunar tide appears in CHAMP data at a period of 13.26 days rather than the actual 14.8 days. Traditionally, Fourier (e.g. Forbes et al., 2008) and least-square (LS; e.g. Azeem et al., 2000) methods are used to study the spatiotemporal characteristics of satellite data. The least-square fitting for spectral analysis has three distinct advantages over the Fourier methods: (1) it can handle unevenly spaced data (2), it provides an estimate of error and (3) it can estimate the amplitude and phase for a specific frequency. In our analysis, we adopt the LS fitting proposed by Wu et al. (1995) to map the EEJ data into the frequency-wavenumber domain. Each EEJ measurements $y_{i}$ can be expressed as

$$
\begin{aligned}
y_{i} & =\sum_{n, s}\left\{A(n, s) \cos \left[2 \pi\left(n t_{i}+s \lambda_{i}\right)\right]\right. \\
& \left.+B(n, s) \sin \left[2 \pi\left(n t_{i}+s \lambda_{i}\right)\right]\right\},
\end{aligned}
$$

where $n$ is the frequency (1/day), $s$ is the wavenumber, $t$ is the normalised time of the day and $\lambda$ the normalised longitude of the measurement. The 2-D coefficients $(A(n, s)$ and $B(n, s))$ are estimated by fitting the above model to the EEJ data. The EEJ time series are typically non-Gaussian due to the non-stationarity of the ionosphere and magnetosphere (for example during a geomagnetic storm), and the standard LS method is not sufficiently protected against the influence of outliers. A few outliers can seriously affect the LS fitting (Sutarno and Vozoff, 1989). We minimise this issue by using an iteratively reweighted LS (IRLS) approach which has been previously applied to derive reliable transfer function estimates from noisy magnetotelluric data (e.g. Chave et al., 1987). Finally, we calculate a $p$ value for each of the coefficients we estimate. The $p$ value represents the probability of getting a coefficient as large as the observed value by random chance. If the $p$ value is small, say less than 0.05 , then the coefficient is regarded as statistically significant. With $10 \mathrm{yr}$ of EEJ observations available for this study, we estimated the amplitude and phases of most of the wave components with this high statistical significance.

We limit our data sampling to the local time sector 07:0017:00 LT, when the EEJ currents are strong enough to generate sensible magnetic signatures at CHAMP altitude. First we scanned the EEJ spectrum in the period range from 3 to $30 \mathrm{~h}$ and for wavenumbers from -10 to 10 to identify the significant wave components in the frequency-wavenumber spectrum. Subsequently, we selected the major components, which are statistically significant, for further studies.

Due to the discrete sampling of the EEJ by CHAMP in time and space certain signals in the frequency-wavenumber spectrum could alias into others. According to Salby (1982) the signals at $\left(n_{1}, s_{1}\right)$ and $\left(n_{2}, s_{2}\right)$ are aliasing to each other if $\left|n_{1}-c s_{1}\right|=\left|n_{2}-c s_{2}\right|$, where $c$ is the shift per day of the satellite orbit through local time ( $c=1$ for a Sunsynchronous satellite and $c=0.996$ for CHAMP). Although $c$ differs only slightly from 1 , we are not facing the problem of aliasing in the considered frequency and wavenumber space. Since we are analysing datasets of 5 or even $10 \mathrm{yr}$ in one run, all the spectral components can be separated uniquely.

To assess the effect of discrete sampling in more detail, we performed two numerical experiments. In the first case a synthetic input signal containing exclusively the migrating diurnal tide DW1 ( $n=1$ and $s=1$ ) was used with unity amplitude and realistic phase. This signal was sampled at the original CHAMP times and locations, i.e. limited to the local time sector 07:00 to 17:00 LT within the years 2005-2010. Then we analysed with our approach the amplitudes and phases of all resulting harmonics of the migrating components. For the second test a synthetic non-migrating tidal component DE3 $(n=1$ and $s=-3$ ) was used with unity amplitude and realistic phase. This signal was again sampled at the original CHAMP times and locations, but limited to the months July through September of all ten years. Results of the two analyses are presented in Sects. 5.1 and 5.2.

\section{Temporal-spatial spectrum of the electrojet}

In the previous section we outlined the approach for determining the complete EEJ spectrum related to solar tides. When interpreting the various components it has to be considered that the electrojet intensity reflects the product of conductivity times driving electric field. Both quantities are influenced by solar tides in different ways; thus spectral components of the EEJ cannot be related one-to-one to tidal components. We will consider this fact in Sect. 5 when it comes to the interpretation of the various waves.

We start with presenting the average observed spectra. To obtain them, data of $5 \mathrm{yr}$ were analysed as one set. Within $5 \mathrm{yr}$ CHAMP provides an even sampling of all local times during each month of a year. Since CHAMP was in orbit for $10 \mathrm{yr}$, we processed two independent $5 \mathrm{yr}$ periods, August 20002005 and August 2005-2010. The first period covers the peak of solar cycle 23, and the second coincides with the deep and prolonged solar minimum. Average solar flux values for the two periods are $F 10.7=145 \pm 44$ sfu and $F 10.7=76 \pm$ $16 \mathrm{sfu}$, respectively.

Tidal spectra from these two independent datasets are shown in Fig. 2. Both spectra exhibit much the same features. Plotted are the EEJ amplitudes at different longitudinal wavenumbers and periods. The spectra are quite patchy. This is mainly due to the clear concentration of the wave power to the harmonics of a diurnal cycle. Appreciable signal can be found up to the eighth harmonic. Higher harmonics are not accessible with a satellite, due to the $90 \mathrm{~min}$ orbital period. Gaps in wavenumber are due to the stringent Student $t$ test. Here we require a $p<0.05$ for a signal to be significant (see Sect. 2 for details). Interestingly, the spectrum from the second, the solar minimum period, is cleaner and more 

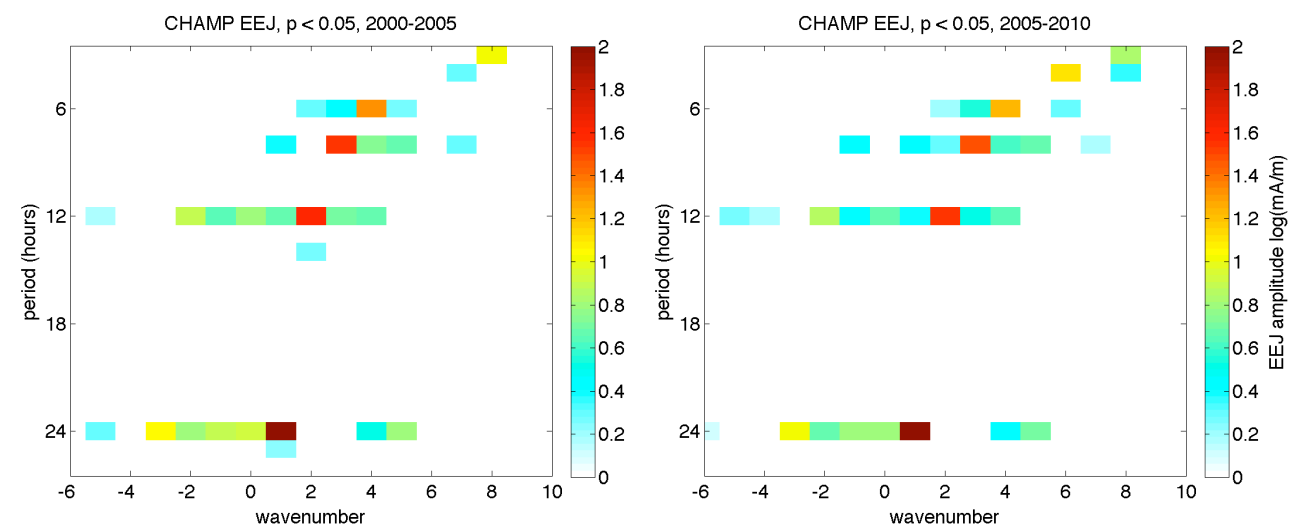

Fig. 2. Average solar tidal spectra of the electrojet for two periods, from solar maximum (left) and solar minimum (right). Colour-coded current densities are shown on a logarithmic scale.

complete. During that time the tidal control of the EEJ seems to be more dominant.

To describe the various spectral components, we use the common tidal terminology. The first capital letter identifies the period; $\mathrm{D}$ stands for diurnal, $\mathrm{S}$ for semi-diurnal, $\mathrm{T}$ for ter-diurnal, Q for quatra-diurnal. The second letter and the subsequent number identify the wavenumber. Negative wavenumbers start with $\mathrm{E}$ (eastward) and positive with $\mathrm{W}$ (westward), then follows the number of wave cycles appearing around the globe. For example, the diurnal westwardmigrating tide is coined DW1. Hereafter we make use of this terminology also for the spectral components of the EEJ although they generally do not represent tides in the classical sense.

As expected, largest amplitudes are found for the migrating components. They are westward propagating, and their wavenumber, $s$, is equal to the harmonics, $n,(s=n)$. The diurnal variation is by far the largest, but higher harmonics also have appreciable amplitudes. Actual results up to the quatra-diurnal are listed in Table 1 for both time intervals. The amplitudes follow approximately a $1 / f$ spectrum, and the EEJ intensity is on average $16 \%$ larger during solar maximum compared to minimum conditions. This ratio is practically the same for all harmonics and is rather moderate when considering the substantial difference in solar flux.

The second-largest amplitude is found for the DE3 component. This is consistent with many previous studies of the EEJ (e.g. Lühr et al., 2008, 2012a, and references therein). It appears as a four-peaked (wave-4) pattern in longitude versus local time plots. This spectral component can be found in satellite observations of almost all thermospheric and ionospheric quantities. In the semi-diurnal harmonics the SE2 is quite strong. This is interesting because it contributes also to the wave-4 pattern. In Sect. 5.2 we will treat the wave-4 signature in more details.

Eastward-propagating tidal components (e.g. DE2 and DE3) are often related to weather phenomena that show
Table 1. Mean amplitudes of the first four harmonics of the EEJ migrating components separately for solar maximum and solar minimum periods. The synthetic signal listed in the two right columns is described in Sect. 5.1.

\begin{tabular}{lrrrrr}
\hline $\begin{array}{l}\text { Migrating } \\
\text { component }\end{array}$ & $\begin{array}{r}2000-2005 \\
{\left[\mathrm{~mA} \mathrm{~m}^{-1}\right]}\end{array}$ & $\begin{array}{r}2005-2010 \\
{\left[\mathrm{~mA} \mathrm{~m}^{-1}\right]}\end{array}$ & Ratio & $\begin{array}{r}\text { Synthetic } \\
{\left[\mathrm{mA} \mathrm{m}^{-1}\right]}\end{array}$ & $\begin{array}{r}\text { Phase } \\
{[\mathrm{h}]}\end{array}$ \\
\hline DW1 & 110 & 94.7 & 1.16 & 95 & 12 \\
SW2 & 41.3 & 36.3 & 1.14 & 38 & 11 \\
TW3 & 35.5 & 31.4 & 1.13 & 24 & 11 \\
QW4 & 21.2 & 17.5 & 1.21 & 14 & 11 \\
\hline
\end{tabular}

local-time-dependent variations (e.g. Hagan and Forbes, 2002). However, from Fig. 2 it is obvious that for higher harmonic waves the westward-propagating components start to dominate. These components have often been explained by an interaction of the migrating tide with stationary planetary waves (e.g. Forbes and Wu, 2006). This point will be revisited in Sect. 5.

In the left frame of Fig. 2 there appears some significant wave power at periods that do not fit diurnal harmonics. They seem to reflect an amplitude modulation of the EEJ migrating tide. A wave patch is found at wavenumber 1 and a period of $25 \mathrm{~h}$. For a periodic modulation of harmonic signals we can write

$A \cos (\Omega t) \cos (\omega t)=\frac{1}{2} A[\cos (\Omega t+\omega t)+\cos (\Omega t-\omega t)]$,

where $\Omega$ reflects the frequency of the modulating signal and $\omega$ that of the harmonic signal. From the $1 \mathrm{~h}$ difference in period to the diurnal variations we obtain a period for the modulation signal of about 25 days. This is close to the solar rotation period of 27 days and reflects probably the influence of the varying solar flux on the EEJ intensity. Similarly, the wave patch at a period of $14 \mathrm{~h}$ and wavenumber 2 can be explained by a 3.5-day modulation of the semidiurnal harmonics. A possible candidate for causing the modulation seems to be the quasi 3-day ultra-fast Kelvin wave 
(e.g. Forbes, 2000). But since the Kelvin wave is eastward propagating with wavenumber 1 , an interaction with the diurnal or ter-diurnal migrating tide is needed to produce the observed wavenumber 2 component. Both these possible products have periods that are way off the observed $14 \mathrm{~h}$. We thus have no immediate explanation for this tiny signal.

During the solar minimum years (right frame) no such sidebands appear. This is in support of our suggestion that tidal forcing of the EEJ is more dominant during quiet times. There appear also some weak signals at large negative wavenumbers. These are probably due to aliasing effects and are not considered any further.

\section{Annual variation of the spectral components}

In the previous section we have presented EEJ wave signals averaged over $5 \mathrm{yr}$ periods. The observed change of the spectra from solar maximum to solar minimum can be regarded as moderate. However, from Fig. 1 we know that the longitudinal distribution of the EEJ intensity is changing a lot with season. Therefore we are now going to investigate the prime spectral components on a month-to-month basis. By this approach we want to recover the annual variation of the spectrum. In order to have enough data available all ten years are taken into account here in one run.

As mentioned before, one has to take into account the various fields that contribute to the EEJ intensity when interpreting the spectrum. In this section we will just present the observed spectral components without providing an in-depth relation to the driving tides.

\subsection{Migrating components}

We start with the largest signals. Figure 3 shows the average annual variation of six prominent diurnal harmonics of the migrating components. The fifth and seventh harmonics are not resolved since these numbers cannot divide into $24 \mathrm{~h}$ evenly. Due to the large range in amplitude a logarithmic scale has been used. It is quite interesting to note that all the harmonics follow the same annual variation pattern. EEJ amplitudes are larger on global average during equinoxes and around December solstice but smallest in June-July. This has been noted before (e.g. Lühr et al., 2008, and references therein). A new observation is the synchronous variation of all the higher harmonics.

To analyse the annual variation of the migrating amplitudes more quantitatively, a harmonic analysis of the monthly values has been performed. Obtained results for the first four diurnal harmonics are listed in Table 2, left part. In order to represent the actual seasonal variations well, annual, semi-annual and ter-annual functions are needed. Annual and semi-annual components have comparable amplitudes for all the migrating harmonics, while the ter-annual is only half as strong. The annual wave peaks early in the

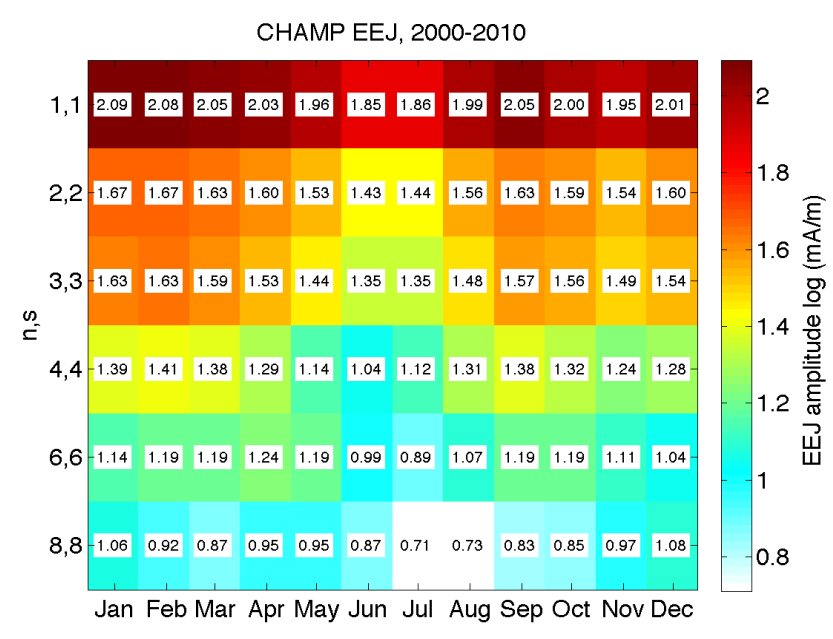

Fig. 3. Annual amplitude variation of electrojet migrating components. Colour-coded current densities are shown on a logarithmic scale.

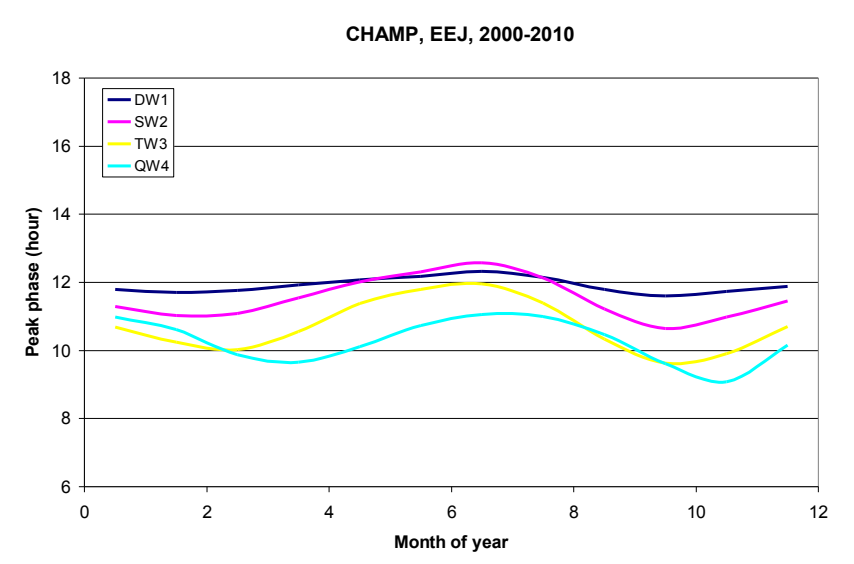

Fig. 4. Annual variations of the phases of the first four harmonics of the EEJ migrating components.

year and the semi-annual shortly before equinoxes. The terannual enhances the January and September peaks. Interestingly, the seasonal peaks appear at similar days of the year (DoY) for all the four diurnal harmonics considered. This confirms again the synchronous variation of the migrating signals.

Also the phases of the migrating components exhibit interesting variations over the year. In the case of the diurnal wave the EEJ amplitude peaks before noon during a major part of the year. Only around June solstice the tidal crest appears some $30 \mathrm{~min}$ after noon. An even stronger modulation of the phase is observed at higher harmonics, as can be seen in Fig. 4. The delayed culmination of the EEJ amplitude around June-July has previously been noticed (e.g. Lühr et al., 2008; see Fig. 3) and may be related to the minimum in amplitude around that season. The phases of the higher harmonics, as shown in Fig. 4, are systematically earlier by 
Table 2. Annual variation of the various harmonics of EEJ migrating components.

\begin{tabular}{lrrrr}
\hline $\begin{array}{l}\text { Annual } \\
\text { harmonics }\end{array}$ & DW1 & SW2 & TW3 & QW4 \\
\hline Mean [mA m $^{-1}$ ] & 101 & 38.6 & 33.9 & 19.7 \\
Annual [mA m $^{-1}$ ] & 16.7 & 6.9 & 7.8 & 4.3 \\
Phase [DoY] & 18 & 17 & 9 & 2 \\
Semi-ann. [mA m & -1 \\
Phase [DoY] & 13.0 & 5.0 & 5.1 & 5.0 \\
Ter-ann. [mA m & -1 \\
Phase [DoY] & 67 & 68 & 68 & 64 \\
& 8.1 & 3.2 & 2.7 & 1.3 \\
& 9 & 10 & 19 & 15 \\
\hline
\end{tabular}

about an hour in local time but follow in principle the same annual variation as the diurnal but with larger amplitudes. We will revisit this issue in the discussion Sect. 5.1.

\subsection{Non-migrating spectral components}

The effect of non-migrating tides on the EEJ has been discussed in a number of previous studies (e.g. England et al., 2006; Lühr et al., 2008, 2012a). Before going into this kind of discussion we first present the complete EEJ spectrum. Figure 5 shows the annual variation of the diurnal non-migrating components. As mentioned before, negative wavenumbers at the ordinate represent eastward-propagating waves and positive westward.

Clearly outstanding in Fig. 5 is the strong DE3 component during the months around August. The DE3 activity is mirrored at lower amplitude by DW5. Both these components contribute to the four-peaked longitudinal structure, which appears as a prominent feature in the September frame of Fig. 1. In the literature (e.g. Pedatella et al., 2008) the DE2 tide also received some attention, in particular with respect to zonal wind and temperature tidal signatures at MLT altitudes. However, in the EEJ spectrum the DE2 amplitude is not outstanding compared to other components. Largest amplitudes are obtained around June solstice. Turning to westward-propagating non-migrating components, we find largest amplitudes for DW3 and to a lesser extend for DW4 around December solstice. The prominence of these components appearing around New Year has previously been noted by Lühr et al. (2012a, Fig. 6). We will revisit these components contributing to wave- 2 and -3 longitudinal patterns in Sects. 5.2 and 5.3.

Figure 6 shows the phases of some of the diurnal nonmigrating tides. Wave crests of DE3 and DE2 pass the Greenwich meridian shortly before and after noon, respectively. These times, as well as the slight annual variation in antiphase with respect to each other, are in line with the report of Lühr et al. (2012a). The phase of DW5 follows closely that of DE2. DW3 stays close to $10 \mathrm{~h}$ around December solstice and is not significant during other times of the year.

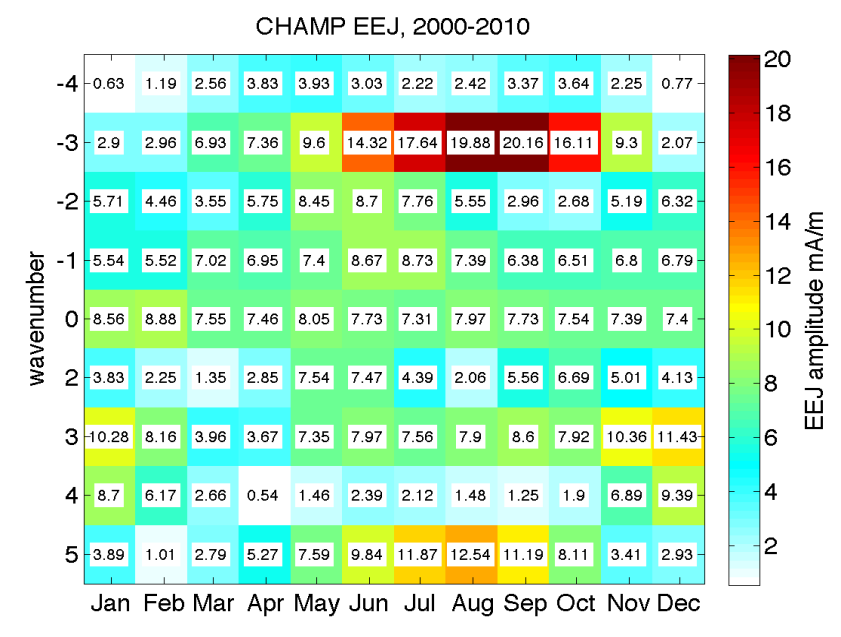

Fig. 5. Annual variation of the diurnal non-migrating EEJ spectral components.

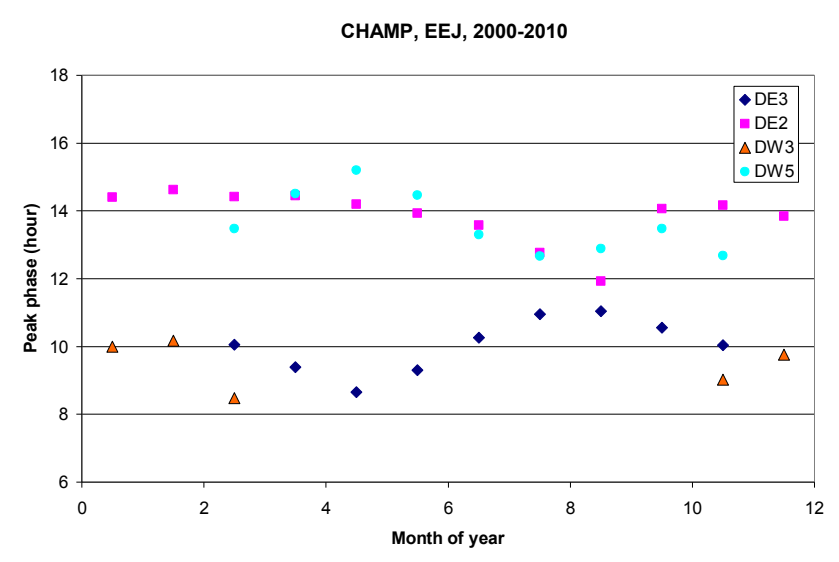

Fig. 6. Annual variation of the phases for some diurnal components. The phase reflects the times when the wave crests of a component passes the Greenwich meridian.

Along the same line of argument we can interpret the semidiurnal non-migrating components shown in Fig. 7. Quite outstanding is the SE2 during summer and fall. This component peaks at the same time of the year as DE3 and contributes also to the four-peaked longitudinal structure. SW4 is strong during the months around December solstice. Pedatella et al. (2008) had identified SW4 as a prominent tidal component in MLT zonal wind data of that season. This component contributes to wave- 2 as DW3 does. Rather interesting is the SW3. Remarkable amplitudes are observed around May and October. This spectral component has previously been found to be particularly strong in the vertical plasma drift over the magnetic equator (e.g. Lühr et al., 2012a). Further discussions of this component follow in Sect. 5.4.

The annual variations of prominent semi-diurnal tidal phases are shown in Fig. 8. It has to be noted that semidiurnal waves exhibit two wave crests separated by $12 \mathrm{~h}$. 


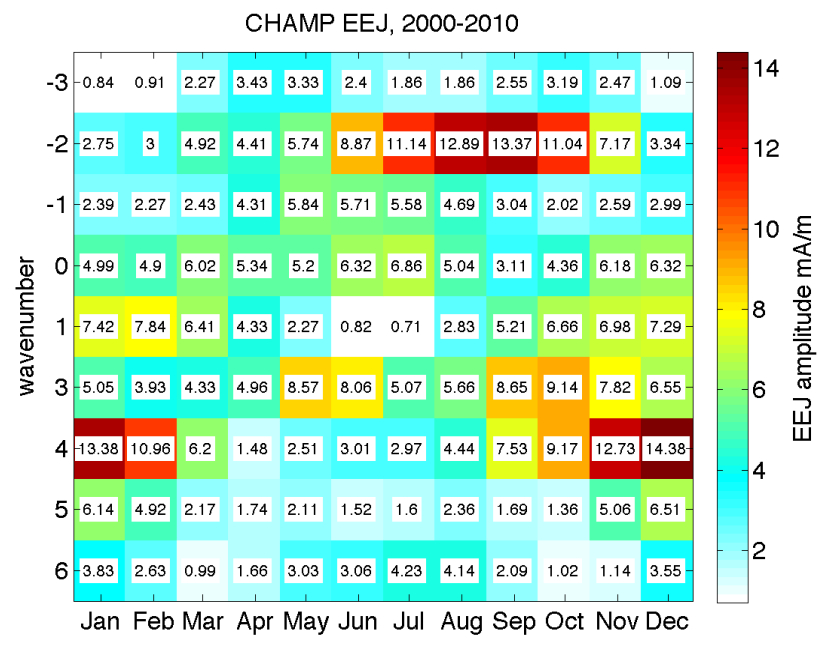

Fig. 7. Same as Fig. 5 but for semi-diurnal components.

CHAMP, EEJ, 2000-2010

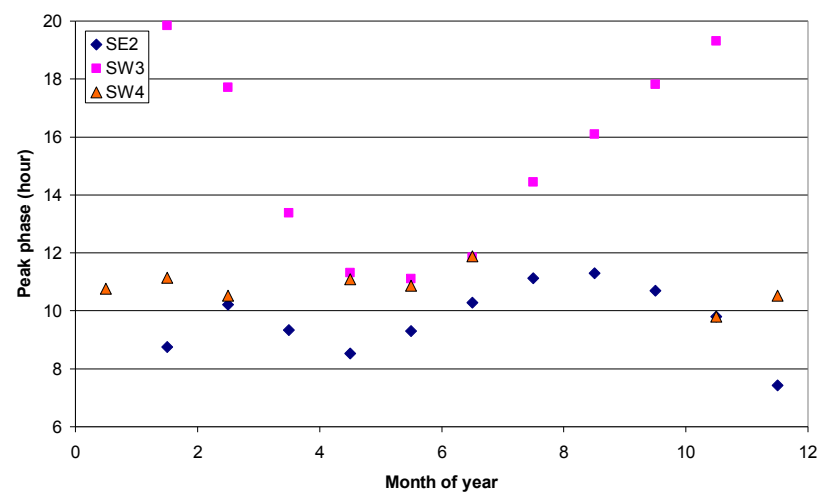

Fig. 8. Same as Fig. 6 but for semi-diurnal phases.

Here we have picked the crest that passes the Greenwich meridian during daytime. The phase of SE2 varies around 10 h quite similar to that of DE3, suggesting the close relation of these two components. Also for the SW4 we find phase values around $10 \mathrm{~h}$ during the prominent December solstice months. Rather interesting is the phase behaviour of SW3. While around May-June the tidal crest passes at about $11 \mathrm{~h}$, we find phase values of $18 \mathrm{~h}$ for October, the other maximum month. This peculiar annual variation of the phase may hint at different drivers being active during the two seasons.

The large CHAMP dataset allows an investigation of even higher diurnal harmonics. Figure 9 presents the annual variations of the non-migrating ter-diurnal components. Quite outstanding is the TW5 during December solstice months. This component also contributes to the wave-2 longitudinal pattern. Furthermore, TW4 has a peak in May-June but is strong through the months to September. This activity may be seen in connection with the SW3 tide. Other

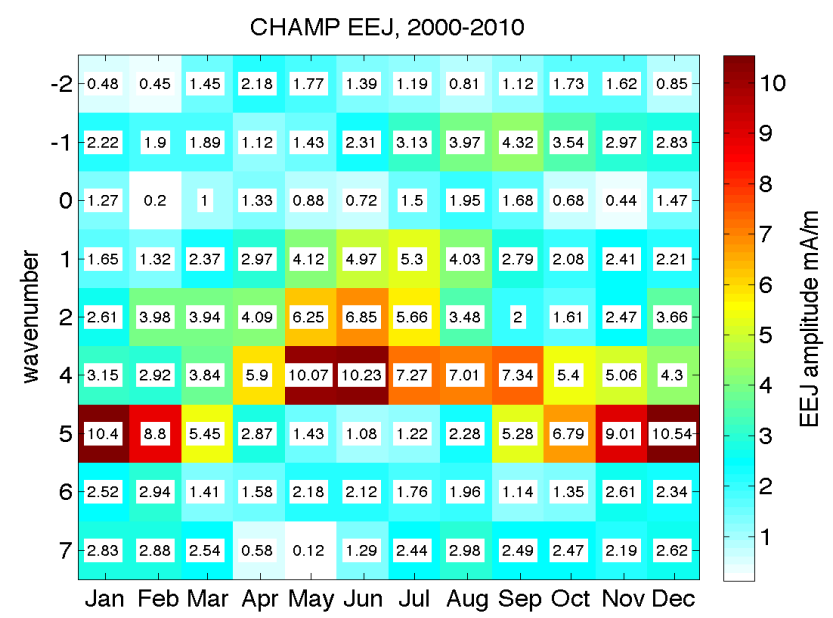

Fig. 9. Same as Fig. 5 but for ter-diurnal components.

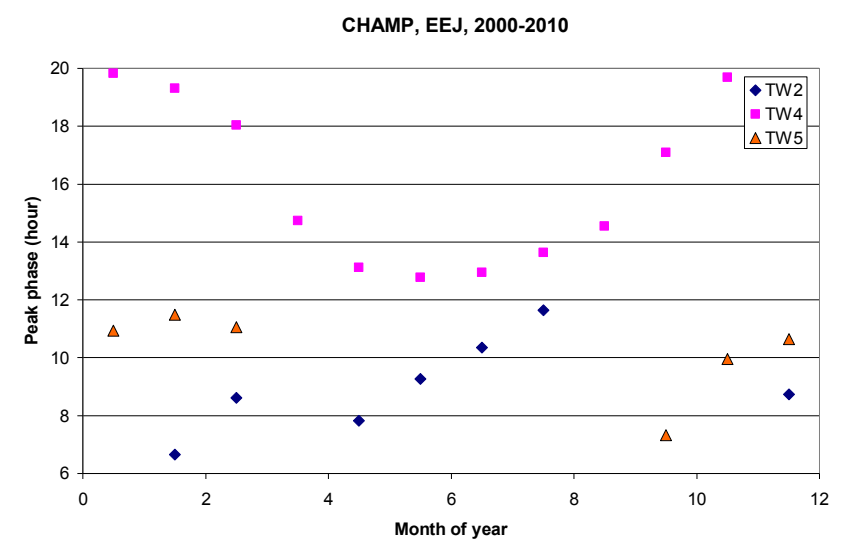

Fig. 10. Same as Fig. 6 but for ter-diurnal phases.

westward-propagating components develop moderate peaks around June solstice.

Annual variations of the phases are depicted in Fig. 10 for the three prominent ter-diurnal components. For TW4 we find a similar phase characteristic to that of SW3, suggesting a relation between these components. In the case of TW5, phase values between $10 \mathrm{~h}$ and $11 \mathrm{~h}$ are observed around December solstice, which is consistent with the results from related diurnal harmonics.

Finally we have looked at the quatra-diurnal components as shown in Fig. 11. Although amplitudes are quite low at that harmonic, the resulting signatures are consistent with the above-presented features. Largest amplitudes appear at May and June for QW3 and QW5. Noteworthy is also the signal strength of QW6 around December solstice. This fits again with the related tidal components at lower harmonics. For the quatra-diurnal components no phases are shown because of the uncertainties expected for these small amplitudes.

Overall, the different spectra of the non-migrating diurnal harmonics give a consistent picture of the EEJ tidal activity. 


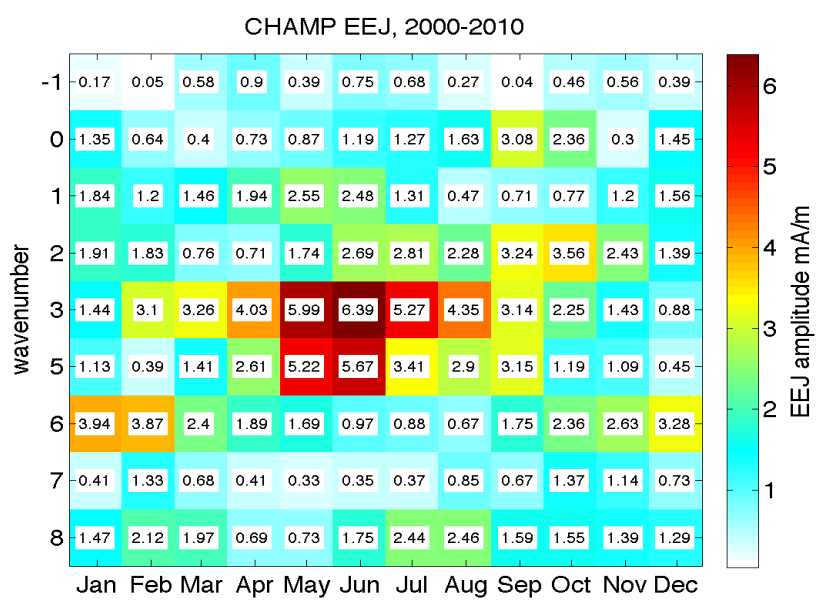

Fig. 11. Same as Fig. 5 but for quatra-diurnal components.

In Sect. 5 we try to present a more comprehensive view of the principal tidal features being responsible for the observed EEJ spectrum by combining related contributions from the different harmonics.

\subsection{Stationary planetary waves}

Third contributors to be considered in the tidal context are the stationary planetary waves. So far their spectrum for the EEJ has never been published. Clearly outstanding is the SPW4 peaking during the months around August as shown in Fig. 12. Its annual variation resembles very much that of DE3 and SE2. This suggests a common source for these spectral components. Stationary planetary waves at other wavenumbers are of quite different character and they peak at solstice seasons. SPW3 exhibits largest amplitudes around December solstice and a secondary maximum around June solstice. In a previous study Lühr et al. (2008) tried to determine the three-peaked stationary longitudinal signature of the daytime EEJ for June and December. They reported 14.9 and $11.8 \mathrm{~mA} \mathrm{~m}^{-1}$ for June and December, respectively. Here we obtain 7.9 and $9.2 \mathrm{~mA} \mathrm{~m}^{-1}$. Both our values are lower than the ones reported previously. One reason is the difference in solar flux level. Lühr et al. (2008) considered the EEJ at $F 10.7=150 \mathrm{sfu}$, while for this study the average value is $F 10.7=110$ sfu. Thus the December results seem to fit. Also the reported longitude of $-53^{\circ}$ for the wave crest for that month is in agreement with our result $\left(-50^{\circ}\right.$; see Fig. 13). On the other hand, the large reported June amplitude is not consistent with the results obtained here and suggests some leakage of non-stationary wave- 3 spectral features into the analysis of Lühr et al. (2008).

The two planetary waves SPW1 and SPW2 are strongest during the months May, June, and July. This may be related to the latitudinal variation of the dip equator over longitude in connection with the sub-solar region in the Northern Hemisphere during those months.

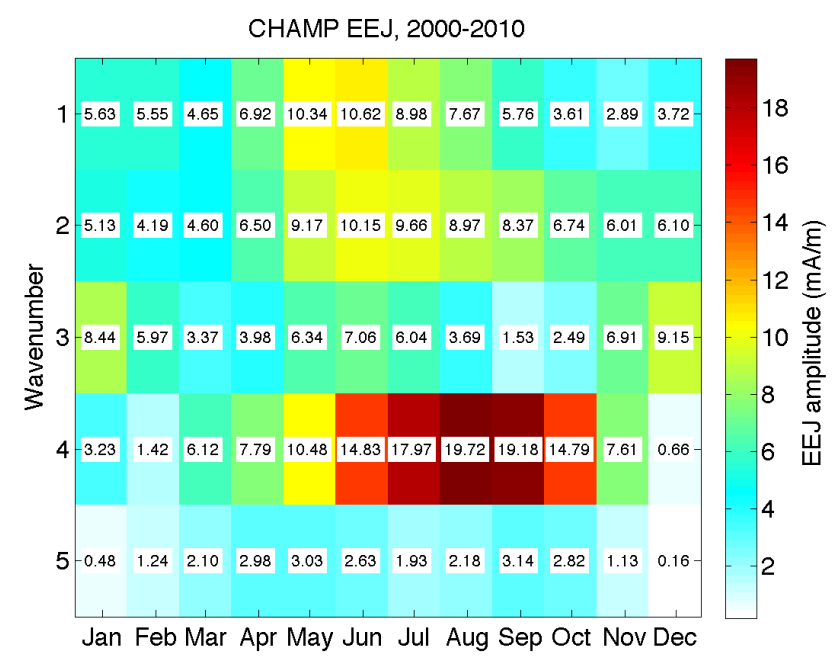

Fig. 12. Annual variation of the EEJ stationary planetary wave amplitudes for the different zonal wavenumbers.

CHAMP, EEJ, 2000-2010

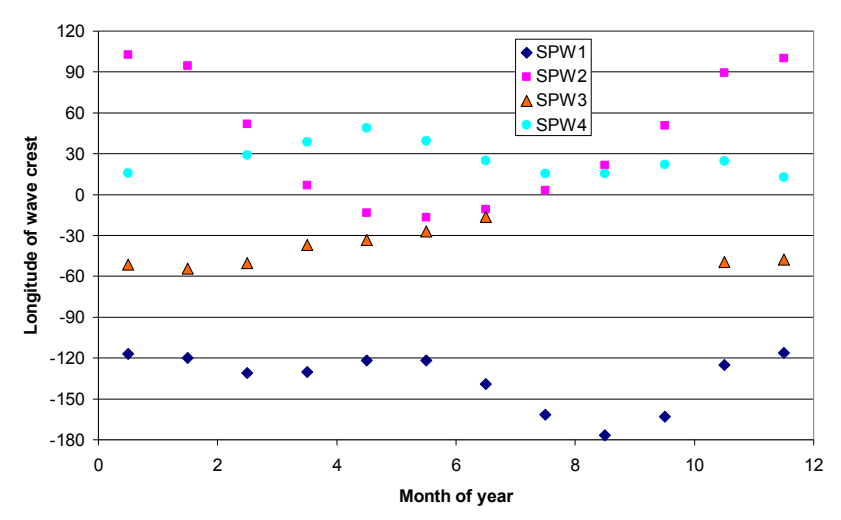

Fig. 13. Annual variation of the longitudes at which the stationary planetary waves peak, separately for the different zonal wavenumbers.

The annual variations of the stationary planetary wave phases are shown in Fig. 13. Presented are the longitudes of the wave crests. For higher zonal wavenumbers the longitude of the crest closest to the Greenwich meridian is shown. In the case of SPW2 the longitudes vary significantly between June and December. One of the two crests moves from western Africa to eastern Asia in that time frame and the second from the central Pacific to the west coast of South America.

\section{Tidal interpretation of the EEJ spectrum}

In the previous sections all the derived spectral components of each diurnal harmonic have been presented. For the physical interpretation of the EEJ characteristics in terms of atmospheric tides we have to consider properly the generation mechanism of the electrojet. Most important in this context are variations of the wind at $E$ layer altitudes producing part of the electric field, $E$, driving the EEJ. The observed 
current density, $j$, is additionally modulated by the conductivity $\sigma: j=\sigma E$ (for more details see Richmond, 1973; Reddy and Devasia, 1981). The diurnal variation of the conductivity can be described by tidal terminology comprising $\sigma_{\mathrm{DW} 1}, \sigma_{\mathrm{SW} 2}$, etc. and the longitudinal variations represented as $\sigma_{\mathrm{SPW} 1}, \sigma_{\mathrm{SPW} 2}$, etc. The effective electric field has its own and independent tidal spectrum. Some ideas of it can be obtained from the vertical plasma drift tidal spectrum derived from ROCSAT 1 data (Lühr et al., 2012a, Fig. 6) or from the non-migrating tidal temperature amplitudes in the equatorial dynamo region (Forbes et al., 2008, Fig. 7). The combined effect of the two quantities ( $E$ field and conductivity) is responsible for the rich spectrum of the EEJ. A proper decomposition of the influences seems to be a rather challenging task.

We have chosen a more practical approach deciphering the tidal influences on the EEJ. As described in Sect. 2, for two examples systematic signals of tidal components were sampled at CHAMP times and positions, and subsequently the resulting spectra analysed. This procedure showed us which additional harmonic components are caused by the day/night contrast of the conductivity. The obtained information is used in the interpretation when combining those spectral components that are related to the same tidal effect.

\subsection{The migrating tides}

These prime tidal components move with the Sun around the Earth. Although they exhibit the largest amplitudes, they have never been studied in detail for the EEJ. The main reason is that they appear in the spectrum of satellite recordings as near-DC signal. Near-polar orbiting satellites precess slowly through local time. Thus base lines have to be stable to deduce reliably the migrating tides from the readings. CHAMP magnetic field measurements provide the required precision for this purpose.

As shown in Fig. 2, migrating components of the EEJ can be observed up to the eighth harmonic. This wide spectrum is mainly caused by the modulation of the migrating tide in the electric field by the day/night contrast of the conductivity. Figure 14 shows the resulting spectrum of a synthetic diurnal migrating tide sampled at CHAMP times and locations during the years 2005-2010. Besides the original signal also higher harmonics than once per day show up. For a quantitative assessment we have listed the results in Table 1 in the two right columns. The amplitudes have been normalised to DW1 of the years 2005-2010, in order to allow for an easy comparison. The synthetic amplitudes are quite similar to those observed, confirming that the diurnal migrating tide of the atmosphere can explain almost completely the migrating spectrum of the EEJ. Just a little addition from the ter-diurnal component seems to be needed. Likewise the phase $11 \mathrm{~h}$ was chosen for the synthetic signal. The phases of the higher harmonics are one hour earlier. This is largely consistent with

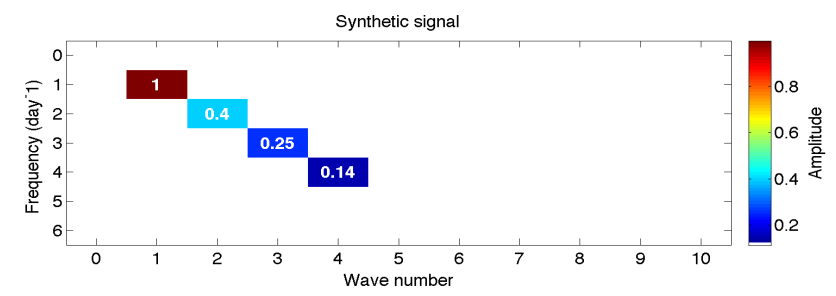

Fig. 14. Spectrum of a synthetic DW1 signal with amplitude 1, sampled at actual times and locations of CHAMP EEJ observations. The spectral harmonics are needed to represent the vanishing signal during the night.

our observations shown in Fig. 4. Also in that case the higher harmonics exhibit earlier phases.

When knowing the origin of the higher harmonics, it is no longer a surprise that all their annual variations are in phase with the diurnal tide. The June-July minimum of the EEJ will be further discussed in Sect. 6 .

\subsection{The wave-4 longitudinal pattern}

It has been recognised since England et al. (2006) that the wavenumber 4 longitudinal pattern is an important feature of the EEJ. During certain parts of the year it dominates the longitudinal variation of the EEJ intensity, as can be seen in the frame for September of Fig. 1. The wave-4 pattern can be made up of contributions from all tidal components for which holds $|s-n|=4$, where $s$ is the zonal wavenumber and $n$ the harmonics of a day. In particular, these are SPW4, DE3, DW5, SE2, SW6, TE1, TW7, Q0, QW8, etc. Many of these spectral components appear in Fig. 2 as highly significant signals of the EEJ. Their amplitudes and phases are listed in Table 3. The values represent averages of the months July through September, when the four-peaked pattern is most prominent. DE3 is the largest, but other components exhibit comparable amplitudes. We want to find out in which way they contribute to the EEJ wave-4 longitudinal pattern.

For this purpose we made a test with synthetic signals. As already mentioned in Sect. 2, the input is a pure DE3 tide which is sampled at the same times and locations as the CHAMP data during the months July through September. Here again, a consequence of signal confinement to daytime is, that a range of other spectral components appears. Figure 15 shows the resulting spectrum of relative amplitudes and phases. Particularly prominent, besides DE3, are the SPW4 and SE2, but higher harmonics are also present. In Table 3 the results from the synthetic signal, now normalised to the actual amplitude of the DE3 tide, are listed in the right half. It is surprising to see how well the values from the synthetic DE3 test match the actual observations (DW5 makes an exception; see below). These results reveal that almost $90 \%$ of the EEJ wave-4 pattern around August can be 


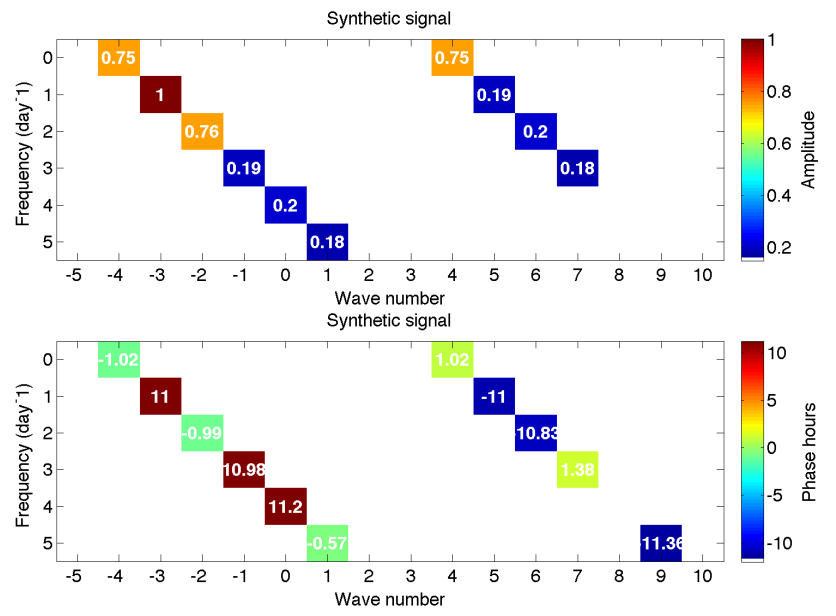

Fig. 15. The same as Fig. 14 but for a synthetic DE3 signal. The lower frame reflects the phase.

explained by the DE3 tide when de-convolving the effects of diurnal ionospheric conductivity modulation.

For a visualisation of the actual EEJ wave-4 pattern we have combined all the observed amplitudes and phases, as listed in Table 3 and plotted the merged signal in Fig. 16. As expected, the well-known pattern emerged during daytime. At night the signal practically vanishes. For reference a dashed line is added marking the phase tilt of a DE3 tide. Except for the pre-noon hours the tilt of the total observed signal is consistent with the DE3 phase front. The phase behaviour at night is considered insignificant, due to the vanishing signal. Thus also the observations confirm the overwhelming dominance of the DE3 tide. The daytime amplitude of the DE3 effect, deduced from Fig. 16, is more than twice as large $\left(\sim 38 \mathrm{~mA} \mathrm{~m}^{-1}\right)$ as the number listed for DE3 in Table 3. All these statements are consistent with previous studies that had limited their attention to EEJ signal from daytime (e.g. Lühr et al., 2008, 2012a).

When comparing the numbers in Table 3 the observed DW5 has significantly larger amplitude than the synthetic component. Also the phase is different from prediction. We think this minor contribution can be generated by an interaction of the migrating tide DW1 with the stationary planetary wave SPW4. The larger observed SPW4 amplitude than predicted suggests that there is also a generic planetary wave. Numerical simulations revealed that a non-linear interaction between DE3 and DW1 at E region altitudes can generate SPW4 (Hagan et al., 2009). Another possibility would be an SPW4 longitudinal pattern of the conductivity, but this is less likely because of its seasonal characteristics; similar conductivity patterns would be expected for spring and autumn.

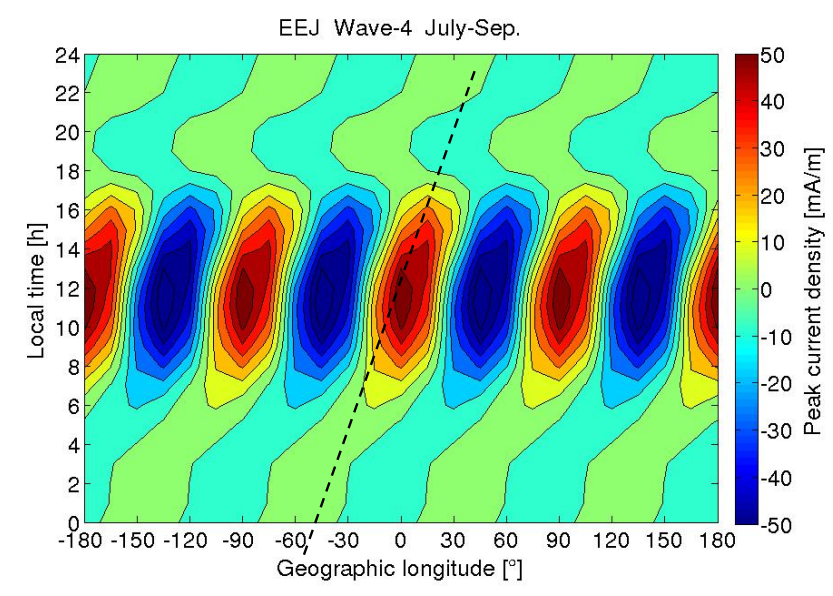

Fig. 16. Composite plot of all the observed spectral components contributing to the wave- 4 longitudinal pattern. The dashed line marks the wave front of a DE3 tide.

The analytical expression describing the interaction between migrating tides and the stationary wave SPW4 is

$$
\begin{aligned}
& A_{n} \cos \left(n \omega t+n \lambda-\varphi_{n}\right) \cos \left(4 \lambda-\varphi_{p}\right) \\
& \quad=1 / 2 A_{n}\left[\cos \left\{n \omega t+(n-4) \lambda-\left(\varphi_{n}-\varphi_{p}\right)\right\}\right. \\
& \left.\quad+\cos \left\{n \omega t+(n+4) \lambda-\left(\varphi_{n}+\varphi_{p}\right)\right\}\right],
\end{aligned}
$$

where $\omega$ corresponds to the one-day period, $n$ is the harmonics of a day, $\lambda$ is the longitude, and $\varphi_{n}$ and $\varphi_{p}$ are the phases of the migrating tides and the planetary wave, respectively. It follows from Eq. (3) that secondary interaction products are expected at wavenumbers $(n-4)$ and $(n+4)$ for the different migrating harmonics. Furthermore, for a given harmonic the amplitudes of the two secondary tidal components should be the same, but the phases, at least in the vicinity of the generation region, are expected to differ by twice the phase of the planetary wave. From a comparison between observed and synthetic signals in Table 3 one may deduce the effect of such a tidal interaction. For example the phase observations of DW5 are in line with the expectations from Eq. (3). According to Table 3 , SPW4 peaks at $17^{\circ} \mathrm{E}$ in longitude. This corresponds to about one hour. As expected, the westwardpropagating tide DW5 has a phase values about $2 \mathrm{~h}$ later than the eastward counterpart, DE3. More research is needed to confirm this suggestion.

There have been reports about significant SE2 contributions to the wave-4 pattern in zonal wind observations at MLT altitudes (e.g. Oberheide et al., 2011). We cannot confirm the effect of a generic SE2 tide from EEJ observations. The SE2 signal deduced here fits in amplitude and phase the spectral content that is needed to represent the DE3 diurnal modulation (see Table 3).

From this detailed analysis we can conclude that the prominent four-peaked longitudinal pattern of the EEJ during the months around August is primarily caused by the DE3 non-migrating tide. The small remaining part can be explained by wave interaction with the diurnal migrating tide. 
Table 3. Mean amplitudes and phases of the EEJ spectral components contributing to the wave-4 longitudinal pattern during the months July to September.

\begin{tabular}{lrrrr}
\hline $\begin{array}{l}\text { Tidal } \\
\text { component } \\
\text { (wave-4) }\end{array}$ & $\begin{array}{r}\text { Amplitude } \\
\text { Jul-Sep } \\
{\left[\mathrm{mA} \mathrm{m}^{-1}\right]}\end{array}$ & $\begin{array}{r}\text { Phase } \\
\text { Jul-Sep } \\
{[\mathrm{h}]}\end{array}$ & $\begin{array}{r}\text { Amplitude } \\
\text { synthetic } \\
{\left[\mathrm{mA} \mathrm{m}^{-1}\right]}\end{array}$ & $\begin{array}{r}\text { Phase } \\
\text { synthetic } \\
{[\mathrm{h}]}\end{array}$ \\
\hline SPW4 & 17.5 & $17^{\circ}, *$ & 14.4 & $15^{\circ}, *$ \\
DE3 & 18.7 & 10.9 & 18.7 & 11 \\
DW5 & 10.6 & 13.0 & 3.9 & 11 \\
SE2 & 12.4 & 11.0 & 14.4 & 11 \\
SW6 & 2.4 & 12.6 & 3.4 & 11 \\
TE1 & 3.9 & 12.2 & 3.9 & 11 \\
TW7 & 2.6 & 10 & 3.2 & 9.2 \\
Q0 & 2.4 & 14 & 3.4 & 11 \\
QW8 & 1.8 & 11 & - & - \\
\hline
\end{tabular}

* In the case of the stationary planetary wave (SPW) the phase value represents the longitude of the wave crest. The two last columns list the signal distribution resulting from the analysis of a synthetic DE3 tide sampled at CHAMP time and location; see text for more details.

\subsection{The wave- 3 longitudinal pattern around June solstice}

Compared to other wavenumbers the wave- 3 pattern is relatively weak in EEJ signal. It develops a peak around June solstice. As suggested earlier, the DE2 non-migrating tide seems to be responsible for it. When taking advantage of the lesson learned about spectral distribution due to signal suppression at night in the case of wave- 4 , we can expect neighbouring sidebands with about $75 \%$ amplitudes. An inspection of the observed sidebands reveals average amplitudes for the months May to July, DE2: $8.3 \mathrm{~mA} \mathrm{~m}^{-1}$, SE1: $5.7 \mathrm{~mA} \mathrm{~m}^{-1}$ and SPW3: $6.5 \mathrm{~mA} \mathrm{~m}^{-1}$. Also the consistent phase, around $14 \mathrm{~h}$, of the three components supports a common origin. When combining the three amplitudes we obtain for the DE2 tidal component a June solstice peak of $12 \mathrm{~mA} \mathrm{~m}^{-1}$, which is fully consistent with the result of Lühr et al. (2012a, Fig. 4).

\subsection{The wave-2 longitudinal pattern around December}

Within the spectra of the various harmonics of non-migrating components the second-largest amplitudes appear around December solstice for westward-propagating waves that contribute to wavenumber 2 . The importance and the origin of this longitudinal structure have never been studied before in detail. Table 4 lists the amplitudes and phases of the related spectral components. Averages of the December and January values have been taken as representative numbers for the effects. The amplitudes are almost comparable with those of the wave- 4 , and the phases of these westward-propagating waves are well aligned.

By combining the contributions of the non-migrating components listed in Table 4 we calculated the corresponding longitudinal pattern (SPW2 has been omitted since it does not fit the concert). Figure 17 shows the resulting longitude
Table 4. Mean spectral amplitudes and phases as in Table 3 but for wave- 2 around December solstice.

\begin{tabular}{lrr}
\hline $\begin{array}{l}\text { Tidal } \\
\text { component } \\
\text { (wave-2) }\end{array}$ & $\begin{array}{r}\text { Amplitude } \\
\text { Dec/Jan } \\
{\left[\mathrm{mA} \mathrm{m}^{-1}\right]}\end{array}$ & $\begin{array}{r}\text { Phase } \\
\text { Dec/Jan } \\
{[\mathrm{h}]}\end{array}$ \\
\hline SPW2 & 5.6 & $101^{\circ, *}$ \\
DW3 & 10.8 & 9.9 \\
SW4 & 13.9 & 10.6 \\
TW5 & 10.5 & 10.8 \\
QW6 & 3.6 & 10.0 \\
\hline
\end{tabular}

versus local time distribution of the EEJ intensity. Double bands of westward-tilted activity stripes appear. For guiding the eye we have over-plotted a dashed line, which represents the tilt of the SW4 phase front. The excellent alignment between dashed line and wave- 2 feature confirms that SW4 is the main contributor to the tidal signature of the EEJ, reaching amplitudes of almost $30 \mathrm{~mA} \mathrm{~m}^{-1}$ around December. Here again we find spectral sidebands with about $75 \%$ amplitude that are needed to facilitate the truncation of the signal to daytime hours. When comparing Fig. 17 to the observations, i.e. the December frame in Fig. 1, similarities are apparent. For example, the westward-tilted EEJ feature, slightly west of the Greenwich meridian, fits well the central positive stripe in Fig. 17. Also the activity maxima on the left and right sides of the frame fit well the resulting wave-2 picture. The resulting SW4 tidal amplitude is almost a factor of 3 larger than the stationary wave- 3 signal which has been quoted by Lühr et al. (2008) as the characteristic EEJ feature around December solstice.

Pedatella et al. (2008) had pointed out the existence of SW4 in zonal wind at MLT altitudes around December solstice, but not stated its dominance nor presented an explanation for its excitation. Also Forbes et al. (2008) identified SW4 as an important non-migrating tidal component in temperature at about $116 \mathrm{~km}$ over the Equator around December solstice. More research is needed to get a better understanding of the generation mechanism of this important component.

\subsection{The wave-1 longitudinal pattern around May-June}

Further remarkable spectral peaks appear around May and June at semi-diurnal and higher harmonics contributing to zonal wavenumber 1 . Possibly contributing components are listed in Table 5. Here the stationary wave SPW1 seems to play an important role for the observed tidal feature. Also in this case we reconstructed the prominent wave- 1 feature by calculating the longitudinal distribution from the combination of SPW1, SW3, TW2, TW4, QW3, and QW5, as listed in Table 5 (DW2 and D0 have been omitted because of incompatible phases). Figure 18 shows the derived composite distribution of the wave-1 EEJ intensity. The longitude 


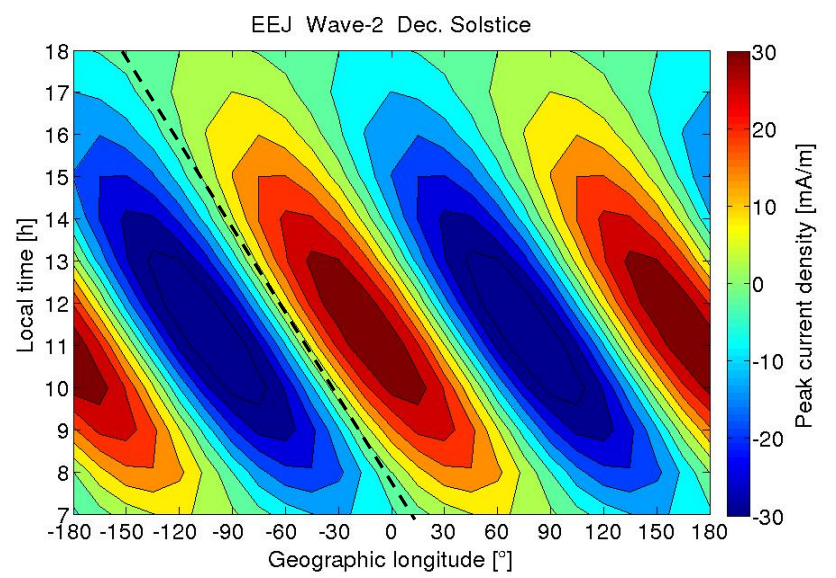

Fig. 17. Composite plot of the major EEJ tidal signals during December solstice.

sector around $120^{\circ} \mathrm{W}$ shows strong enhancements, and the sector around $60^{\circ} \mathrm{E}$ depletions. These longitudes coincide with the SPW 1 crest and trough, respectively. The additional non-migrating components cause an enforcement of the EEJ around 15:00 LT and the truncation of the EEJ to daytime hours. When comparing Fig. 18 with the June frame of Fig. 1 we find certain similarities. Quite prominent in Fig. 1 is the westward-tilted feature of high EEJ intensity up to hours beyond 16:00 LT in the $120^{\circ} \mathrm{W}$ longitude sector. Also a general strengthening of the EEJ in this sector is in line with our reconstruction. Similarly, we can explain the observed counterelectrojets at morning and evening times over Africa by the wave- 1 trough in the sector around $60^{\circ} \mathrm{E}$.

The tidal features responsible for wave-1 during the months around May-June have never been discussed in detail in the literature. Lühr et al. (2012a) mentioned that a prominent wave- 1 feature is also found in the vertical plasma drift. There it has been related to SW3 with peak amplitude in April but staying prominent through September.

From our comprehensive analysis we can deduce that the two non-migrating tides SW3 and TW4 are both viable candidates that may be responsible for the westward-tilted wave1 feature. Both components exhibit the same peculiar annual phase variations (see Figs. 8 and 10). Judging from the amplitudes TW4 should be the responsible tidal component for the May-June EEJ signature, and SW3 and QW3 just the required sidebands. We are not aware of a previous mention of TW4 as a prominent tidal component at $\mathrm{E}$ region altitude around early summer. Our preferred explanation for TW4 is an interaction of the migrating tide TW3 with the standing wave SPW1. We suggest that SWP1 is related to the latitude variation of the magnetic equator with longitude. Later in the year the role of the two components seems to switch. From October through December SW3 is dominating and TW4 is considered a sideband effect. This inference of SW3 being a prominent upper-atmospheric tide around the end of the year

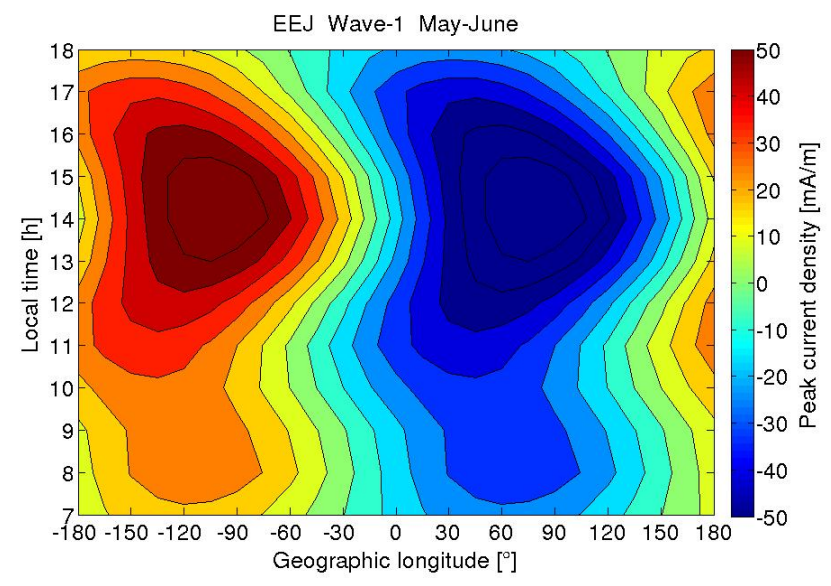

Fig. 18. Composite plot of the prominent wave-1 EEJ tidal signal during May-June.

is in line with observations reported by Lühr et al. (2012a) for the EEJ and the ionisation anomaly and by Forbes et al. (2008) for the temperature. Further details of SW3 will be discussed in Sect. 6 .

\section{Discussion}

In this study we present a full decomposition of the average EEJ signal spectrum related to solar tides. The obtained spectral resolution can only be derived from a long $(10 \mathrm{yr})$ and continuous global coverage of the EEJ intensity, as provided by the CHAMP satellite. We are aware of the fact that in this case any information about temporal variations of tidal signals related to phenomena like Quasi-Biannual Oscillation (QBO) or El Niño Southern Oscillation (ENSO) gets lost (e.g. Häusler et al., 2013). Furthermore, this study differs from most others that focus on tidal effects of ionospheric quantities. They commonly limit their attention to daytime hours when the $\mathrm{E}$ layer conductivity is high. We prefer to determine the full spectrum of the EEJ without making assumptions. The penalty for that is that we get a wide response spectrum that contains a lot of leakage from the driving tidal frequency into sidebands because of the disappearing EEJ signal at night. The spectrum has to be decomposed in order to achieve reliable information about the driving tides. Because of the completeness of our approach we think it provides unprecedented results.

The most prominent feature in the EEJ spectrum is the migrating tide. In previous studies this has generally been neglected. Here we resolved the spectrum up to the eighth diurnal harmonics. The amplitudes fall off approximately as $1 / f$, and all harmonics peak around noontime. Since the EEJ develops appreciable amplitudes only during daytime, the $1 / f$ spectrum is a logical consequence of its characteristic. By analysing a synthetic diurnal tide with our approach 
Table 5. Same as Table 4 but for wave-1 around May-June.

\begin{tabular}{lrr}
\hline $\begin{array}{l}\text { Tidal } \\
\text { component } \\
\text { (wave-1) }\end{array}$ & $\begin{array}{r}\text { Amplitude } \\
\text { May-June } \\
{\left[\mathrm{mA} \mathrm{m}^{-1}\right]}\end{array}$ & $\begin{array}{r}\text { Phase } \\
\text { May-June } \\
{[\mathrm{h}]}\end{array}$ \\
\hline SPW1 & 10.5 & $-122^{\circ}, *$ \\
D0 & 7.9 & 21.1 \\
DW2 & 7.5 & 6.4 \\
SW1 & 1.5 & - \\
SW3 & 8.3 & 11.2 \\
TW2 & 6.6 & 8.5 \\
TW4 & 10.2 & 12.9 \\
QW3 & 6.2 & 14.6 \\
QW5 & 5.4 & 14.4 \\
\hline
\end{tabular}

we can show that the observed spectrum is a consequence of modulating the migrating diurnal tide by the ionospheric conductivity. For that reason it is no surprise that we find synchronous annual variations of all the spectral harmonics. Also the phases of the harmonics vary in much the same way over a year.

The EEJ migrating tidal response shows a clear seasonal variation. Amplitudes are large during December solstice months and the two equinoxes, but they are significantly smaller around June solstice. This EEJ depression during the middle of the year has been recognised already long ago. The so-called "July minimum" is a well-known feature of the ionosphere-thermosphere system (e.g. Rishbeth and MüllerWodarg, 2006). It affects both the conductivity and the driving electric field, but there is still no conclusive explanation for this global annual variation. The ratio between the largest tidal amplitude in January and the smallest in June is 1.7. For comparison, Müller et al. (2009) report for the low-latitude thermospheric density at $400 \mathrm{~km}$ a January-to-July ratio of 1.6. This seems to be a typical value for the annual variation of upper-atmospheric quantities.

The phase of the migrating tide also shows an annual variation. EEJ peak amplitudes are commonly reached before noon but somewhat later around June solstice. In our view this is caused by the prominent TW4 tidal signature (see Sect. 5.3), thus a consequence of the late EEJ maxima around 15:00 LT in the sector west of $90^{\circ} \mathrm{W}$ during that season. The cause of this tidal component would be worth studying in more detail.

We have compared the EEJ amplitude of the migrating tide from periods of high and low solar activity. The obtained difference is around $16 \%$. This value is quite small compared to the substantial difference in solar flux between the two periods ( $F$ 10.7: 145 and $76 \mathrm{sfu}$ ). A possible explanation for the small change in amplitude could be the enhanced magnetic activity during the first analysis period. It is known that magnetic perturbations tend to suppress the EEJ amplitude, while during the solar minimum years the EEJ can fully develop. The gain in ionospheric conductivity from higher solar flux
CHAMP, EEJ tide, 2000/05 and 2005/10

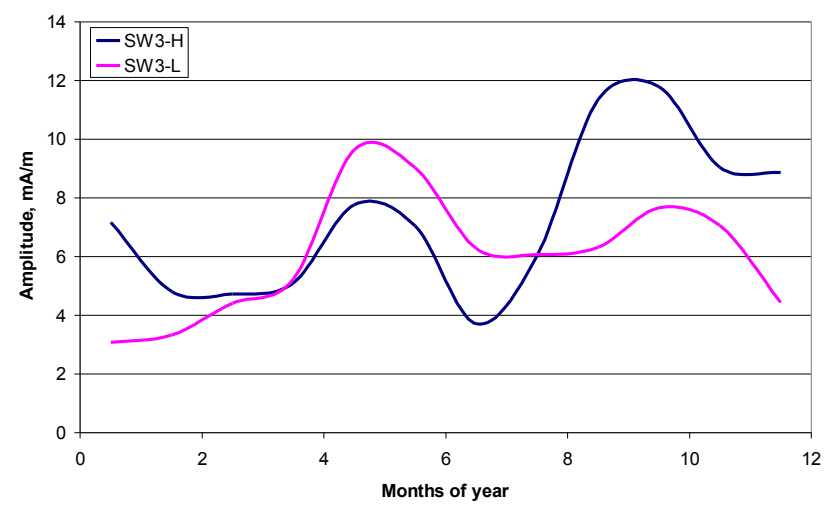

Fig. 19. Annual variations of the SW3 spectral component for periods of high (black) and low (red) solar activity. Amplitudes at spring and autumn respond differently to the change in solar flux level.

may partly be compensated by the larger magnetic activity during solar maximum years (see Kikuchi et al., 2003, 2008).

Almost all the EEJ spectral components exhibit more or less the same solar cycle dependence with an amplitude decrease of $10 \%$ to $20 \%$ from solar maximum to minimum. An exception to that is SW3. This component shows quite different curves for the annual amplitude variations derived from the two periods, 2000-2005 and 2005-2010. As can be seen in Fig. 19, it exhibits two amplitude maxima, around spring and autumn. While the spring peak is part of the response to the TW4 tide (see Sect. 5.3), we relate the amplitude variation around autumn to an activity dependence of the SW3 tide. The tidal amplitude is much enhanced during the period of high solar flux compared to the solar minimum years around the end of the year. Xiong and Lüh (2013) reported similar results about the SW3 tidal effect on the intensity of the equatorial ionisation anomaly. They also find a strong dependence of SW3 on solar activity during the later months of the year but no dependence on magnetic activity. Enhancement of solar flux increases the coupling between ions and neutrals at $\mathrm{F}$ region altitudes. We thus favour an upper-atmospheric driver for this tidal component. More research is needed to disclose the processes responsible for the SW3 non-migrating tidal signal in ionospheric parameters.

The electrojet is affected by a rich spectrum of nonmigrating tides. For this reason the longitudinal distribution of the EEJ intensity varies from month to month (see Fig. 1). It thus seems needless to specify a typical longitudinal pattern of the EEJ. However, the overall tidal activity is not constant throughout the year. Figure 20 shows the root of the summed wave power over all wavenumbers, separately for the different diurnal harmonics. All harmonics show a minimum around March equinox. The wave power maxima appear at quite different times of the year for the different harmonics. We clearly can relate the wave power maxima 
CHAMP, EEJ, 2000-2010

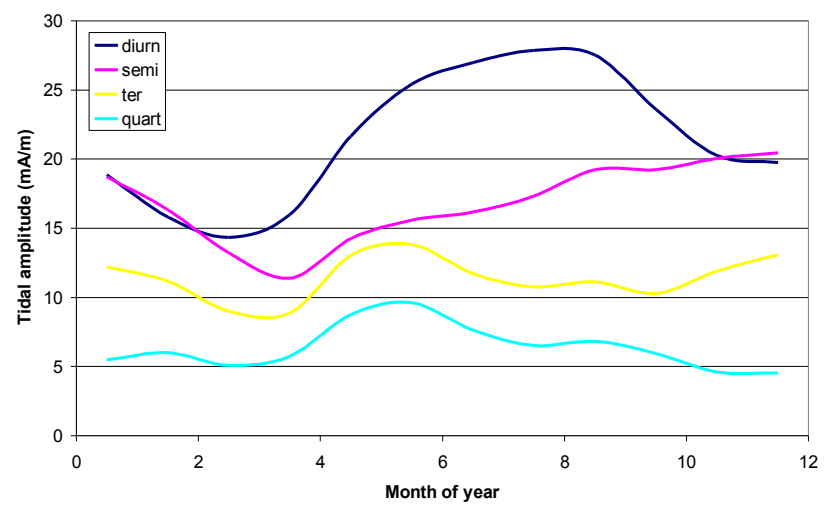

Fig. 20. Annual variation of non-migrating spectral wave power amplitude, separately for the different diurnal harmonics.

to the non-migrating tidal features identified in Sect. 5. The diurnal tides are strongly dominated by the DE3 contribution maximising around August. The sum of semi-diurnal components is largest around December when SW4 and to a lesser extend SW3 dominate the tidal signal. The ter- and quatra-diurnal components make important contribution to wave-1 through TW4 in May-June. Figure 20 demonstrates that smallest longitudinal structures of the EEJ are expected during March. This inference can be confirmed by comparison with the March frames in Fig. 1. During equinox seasons the Sun is over the Equator; thus both hemispheres are expected to be excited most symmetrically. Due to that reason a smooth longitudinal distribution would have been expected also for September equinox. But the strong influence from deep tropical convection in the troposphere during late summer and autumn imposes the wave-4 longitudinal structure. It is probably just the fortunate coincidence of the prominent tropospheric driving with the quiet equinox conditions of the EEJ that the wave-4 structure appears so clearly as a longitudinal modulation of the EEJ intensity (see September frame of Fig. 1). Besides DE3 also DE2 is thought to be excited by deep convection in the tropical troposphere (e.g. Hagan and Forbes, 2002). Since it maximises around June solstice, it appears in competition with other tidal excitations (mainly westward propagating) and is therefore much less obvious in EEJ longitudinal patterns.

At several occasions it has been shown that low-latitude zonal winds around $105 \mathrm{~km}$ altitude share very similar tidal characteristics with the daytime EEJ (e.g. Lühr et al., 2012a; Häusler et al., 2013). For that reason it is suggested that similar analyses should be performed with wind data, e.g. from TIDI, in order to enable a one-to-one comparison between these two related quantities. This may help to interpret the presented tidal spectra and to better understand the coupling mechanisms in the E layer from the neutral atmospheric motion into the ionospheric electrodynamics.

\section{$7 \quad$ Summary and conclusions}

The quiet-time equatorial electrojet is primarily a phenomenon driven by tidal winds in the ionospheric $\mathrm{E}$ region. Here we have presented for the first time the average full spectrum of the EEJ deduced from $10 \mathrm{yr}$ of CHAMP observations. Its knowledge is among others a pre-requisite for the proper interpretation of the non-cyclic effects influencing the EEJ. Major results presented here are summarised below.

1. As expected, the diurnal migrating tide excites the largest amplitude. It causes the mean diurnal variation of the EEJ. Spectral harmonics up to the eighth diurnal harmonic have been observed. Their amplitudes fall off approximately as $1 / f$. Such a spectrum is needed to represent the EEJ activity variation between day- and nighttime hours.

2. The migrating tidal signal shows distinct intra-annual variations. Large amplitudes appear around December solstice and equinox seasons. Significantly smaller amplitudes are observed around June solstice. The ratio between largest amplitudes in January and smallest in June amounts to 1.7. The annual variations of the EEJ tidal amplitude can well be described by harmonic functions including annual, semi-annual and ter-annual terms.

3. The EEJ exhibits a rich spectrum of non-migrating components. Detailed investigations have been performed including the diurnal up to quatra-diurnal spectral components. Strongest is the diurnal eastward-propagating DE3, but also other prominent spectral features emerge. On a month-to-month basis we show the amplitude variations at the various wavenumbers. Each harmonic and wavenumber exhibits its individual annual variation.

4. Besides the tides also stationary planetary waves modulate the EEJ intensity. Their effects up to zonal wavenumber 5 have been analysed. Largest amplitudes for generic planetary waves are found around solstice seasons.

5. The most prominent EEJ tidal feature is the four-peaked longitudinal pattern appearing around August. Our analysis shows that almost $90 \%$ of the signal can be related to the DE3 tide. Deep tropospheric convection in the troposphere is primarily responsible for the DE3. To a lesser extend the DW5 contributes also to the wave-4 pattern. This tide is suggested to be a product of the interaction between the migrating tide DW1 and the planetary wave SPW4. There is no indication in the EEJ signal of a direct SE2 tidal driving.

6. The dominating feature during December solstice months is a wave- 2 longitudinal pattern. The primary tidal component responsible for it is SW4. The mechanism exciting this tide is presently not known, but SW4 
is also found to be prominent in MLT zonal wind and temperature variations around December solstice (e.g. Pedatella et al., 2008; Forbes et al., 2008).

7. The wave-1 longitudinal structure shows an interesting variation over the year being influenced by several tidal components. There appears a prominent stationary wave SPW1 during the months around June solstice, which supports a strong EEJ in the Western Hemisphere and causes a suppression of intensity in the eastern part. In addition to that, a TW4 tide maximises around MayJune, causing the EEJ to develop peak current densities only after 15:00 local time in the Western Hemisphere. During the late months of the year the SW3 tide is the prime contributor to wave-1. It supports the formation of counter-electrojets in the longitude sector around $180^{\circ}$ (see Fig. 1, December frame). The origin of all these tidal components is still not well understood.

8. The semi-diurnal tide SW3 shows a clear dependence on solar flux level in the autumn/winter time frame. Tidal effects of the EEJ almost double when the flux level rises from $F 10.7=76$ to $145 \mathrm{sfu}$. Conversely, this component shows no dependence on magnetic activity. For no other non-migrating tide has such behaviour been found.

This complete EEJ spectrum related to average solar tidal forcing offers the possibility to construct a mean tidal model that represents the cyclically varying part of the EEJ. This model would describe the quiet-time behaviour of the EEJ. It could serve as a reference for investigating the EEJ day-today variability or magnetic disturbance effects.

The actually derived values for all the EEJ spectra are made available at the following websites: http:// www.gfz-potsdam.de/CHAMP_currents and http://geomag. org/models/EEJ/eejspectra.html.

Acknowledgements. The authors thank S. Maus for providing the electrojet intensities derived from CHAMP magnetic field observations. The CHAMP mission was sponsored by the Space Agency of the German Aerospace Center (DLR) through funds of the Federal Ministry of Economics and Technology.

The service charges for this open access publication have been covered by a Research Centre of the Helmholtz Association.

Topical Editor K. Hosokawa thanks S. England and one anonymous referee for their help in evaluating this paper.

\section{References}

Alken, P. and Maus, S.: Spatio-temporal characterization of the equatorial electrojet from CHAMP, Ørsted, and SACC satellite measurements, J. Geophys. Res., 112, A09305, doi:10.1029/2007JA012524, 2007.
Alken, P. and Maus, S.: Electric fields in the equatorial ionosphere derived from CHAMP satellite magnetic field measurements, J. Atmos. Sol.-Terr. Phy., 72, 319-326, doi:10.1016/j.jastp.2009.02.006, 2010.

Azeem, S. M. I., Killeen, T. L., Johnson, R. M., Wu, Q., and Gell, D. A.: Space-time analysis of TIMED Doppler Interferometer (TIDI) measurements, Geophys. Res. Lett., 27, 3297-3300, 2000.

Bartels, J. and Johnston, H. F.: Geomagnetic tides in horizontal intensity at Huancayo, Part I, terrestrial magnetism and atmospheric electricity, J. Geophys. Res., 45, 269-308, 1940.

Chave, A. D., Thomson, D. J., and Ander, M. E.: On the robust estimation of power spectra, coherences, and transfer functions, J. Geophys. Res, 92, 633-648, 1987.

Cowling, T.: The electrical conductivity of an ionized gas in the presence of a magnetic field, Mon. Not. R. Astron. Soc., 93, 9098, 1933.

England, S. L., Maus, S., Immel, T. L., and Mende, S. B.: Longitude variation of the E-region electric fields caused by atmospheric tides, Geophys. Res. Lett., 33, L21105, doi:10.1029/2006GL027465, 2006.

England, S. L., Immel, T. J., Huba, J. D., Hagan, M. E., Maute, A., and DeMajistre, R.: Modeling of multiple effects of atmospheric tides on the ionosphere: An examination of possible coupling mechanisms responsible for the longitudinal structure of the equatorial ionosphere, J. Geophys. Res., 115, A05308, doi:10.1029/2009JA014894, 2010.

Forbes, J. M.: Wave coupling between the lower and upper atmosphere: Case study of an ultra-fast Kelvin wave, Special Jubilee Issue of J. Atmos. Solar-Terr. Phy., 62, 1603-1622, 2000.

Forbes, J. M. and Wu, D.: Solar tides as revealed by measurements of mesosphere temperature by the MLS experiment on UARS, J. Atmos. Sci., 63, 1776-1797, 2006.

Forbes, J. M., Zhang, X., Palo, S., Russell, J., Mertens, C. J., and Mlynczak, M.: Tidal variability in the ionospheric dynamo region, J. Geophys. Res. 113, A02310, doi:10.1029/2007JA012737, 2008.

Hagan, M. E. and Forbes, J. M.: Migrating and nonmigrating diurnal tides in the middle and upper atmosphere excited by tropospheric latent heat release, J. Geophys. Res. 107, D244754, doi:10.1029/2001JD001236, 2002.

Hagan, M. E., Maute, A., and Roble, R. G.: Tropospheric tidal effects on the middle and upper atmosphere, J. Geophys. Res., 114, A01302, doi:10.1029/2008JA013637, 2009.

Häusler, K. and Lühr, H.: Nonmigrating tidal signals in the upper thermospheric zonal wind at equatorial latitudes as observed by CHAMP, Ann. Geophys., 27, 2643-2652, doi:10.5194/angeo27-2643-2009, 2009.

Häusler, K., Oberheide, J., Lühr, H., and Koppmann, R.: The Geospace Response to Nonmigrating Tides, in: Climate And Weather of the Sun-Earth System (CAWSES): Highlights from a priority program, edited by: Lübken, F.-J., 481-506, Springer, Dodrecht, the Netherlands, 2013.

Kikuchi, T., Hashimoto, K. K., Kitamura, T.-I., Tachihara, H., and Fejer, B.: Equatorial counterelectrojets during substorms, J. Geophys. Res., 108, 1406, doi:10.1029/2003JA009915, 2003.

Kikuchi, T., Hashimoto, K. K., and Nozaki, K.: Penetration of magnetospheric electric fields to the equator during a geomagnetic storm, J. Geophys. Res., 113, A06214, 
doi:10.1029/2007JA012628, 2008.

Lühr, H., Rother, M., Maus, S., Mai, W., and Cooke, D.: The diamagnetic effect of the equatorial Appleton anomaly: Its characteristics and impact on geomagnetic field modelling, Geophys. Res. Lett., 30, 1906, doi:10.1029/2003GL017407, 2003.

Lühr, H., Maus, S., and Rother, M.: The noon-time equatorial electrojet, its spatial features as determined by the CHAMP satellite, J. Geophys. Res., 109, A01306, doi:10.1029/2002JA009656, 2004.

Lühr, H., Rother, M., Häusler, K., Alken, P., and Maus, S.: The influence of non-migrating tides on the longitudinal variation of the equatorial electrojet, J. Geophys. Res., 113, A08313, doi:10.1029/2008JA013064, 2008.

Lühr, H., Rother, M., Häusler, K., Fejer, B., and Alken, P.: Direct comparison of non-migrating tidal signatures in the electrojet, vertical plasma drift and equatorial ionization anomaly, J. Atmos. Sol.-Terr. Phy., 75-76, 31-43, doi:10.1016/j.jastp.2011.07.009, 2012a.

Lühr, H., Siddiqui, T. A., and Maus, S.: Global characteristics of the lunar tidal modulation of the equatorial electrojet derived from CHAMP observations, Ann. Geophys., 30, 527-536, doi:10.5194/angeo-30-527-2012, 2012 b.

Manoj, C., Maus, S., Lühr, H., and Alken, P.: Penetration characteristics of the interplanetary electric field to the daytime equatorial ionosphere, J. Geophys. Res., 113, A12310, doi:10.1029/2008JA013381, 2008.

Maus, S., Alken, P., and Lühr, H.: Electric fields and zonal winds in the equatorial ionosphere inferred from CHAMP satellite magnetic measurements, Geophys. Res. Lett., 34, L23102, doi:10.1029/2007GL030859, 2007.

Müller, S., Lühr, H., and Rentz, S.: Solar and magnetospheric forcing of the low latitude thermospheric mass density as observed by CHAMP, Ann. Geophys., 27, 2087-2099, doi:10.5194/angeo27-2087-2009, 2009.

Oberheide, J., Wu, Q., Killeen, T. L., Hagan, M. E., and Roble, R. G.: Diurnal nonmigrating tides from TIMED Doppler Interferometer wind data: monthly climatologies and seasonal variations, J. Geophys. Res., 111, A10S03, doi:10.1029/2005JA011491, 2006.

Oberheide, J., Forbes, J. M., Zhang, X., and Bruinsma, S. L.: Wave-driven variability in the ionosphere-thermospheremesosphere system from TIMED observations: What contributes to the "wave 4"?, J. Geophys. Res., 116, A01306, doi:10.1029/2010JA015911, 2011.

Park, J., Lühr, H., Kunze, M., Fejer, B. G., and Min, K. W.: Effect of sudden stratospheric warming on lunar tidal modulation of the equatorial electrojet, J. Geophys. Res., 117, A03306, doi:10.1029/2011JA017351, 2012.
Pedatella, N. M., Forbes, J. M., and Oberheide, J.: Intra-annual variability of the low-latitude ionosphere due to nonmigrating tides, Geophys. Res. Lett., 35, L18104, doi:10.1029/2008GL035332, 2008.

Rastogi, R. G. and Trivedi, N. B.: Luni-solar tides in H at stations within the equatorial electrojet, Planet Space Sci., 18, 367-377, 1970.

Reddy, C. A. and Devasia, C. V.: Height and latitude structure of electric fields and currents due to local eastwest winds in the equatorial electrojet, J. Geophys. Res., 86, 5751-5767, 1981.

Reigber, C., Lühr, H., and Schwintzer, P.: CHAMP mission status, Adv. Space Res., 30, 129-134, 2002.

Richmond, A. D.: Equatorial electrojet-1. Development of a model including winds and instabilities, J. Atmos.-Terr. Phy., 35, 10831103, 1973.

Rishbeth, H. and Müller-Wodarg, I. C. F.: Why is there more ionosphere in January than in July? The annual asymmetry in the F2-layer, Ann. Geophys., 24, 3293-3311, doi:10.5194/angeo-243293-2006, 2006.

Ritter, P., Lühr, H., Viljanen, A., Amm, O., Pulkkinen, A., and Sillanpää, I.: Ionospheric currents estimated simultaneously from CHAMP satelliteand IMAGE ground-based magnetic field measurements: a statisticalstudy at auroral latitudes, Ann. Geophys., 22, 417-430, doi:10.5194/angeo-22-417-2004, 2004.

Salby, M. L.: Sampling theory for asynoptic satellite observations. Part I: Space-time spectra, resolution, and aliasing, J. Atmos. Sci., 39, 2577-2600, 1982.

Sugiura, M. and Poros, D. J.: An improved Model Equatorial Electrojet with a meridional current system, J. Geophys. Res., 74, 4025-4034, 1969.

Sutarno, D. and Vozoff, K.: Robust M-estimation of magnetotelluric impedance tensors, Explor. Geophys., 20, 383-398, 1989.

Wu, D. L., Hays, P. B., and Skinner, W. R.: A least squares method for spectral analysis of space-time series, J. Atmos. Sci., 52, 3501-3511, 1995.

Xiong, C. and Lühr, H.: Nonmigrating tidal signatures in the magnitude and the inter-hemispheric asymmetry of the equatorial ionization anomaly, Ann. Geophys., 31, 1115-1130, doi:10.5194/angeo-31-1115-2013, 2013.

Zhang, X., Forbes, J. M., and Hagan, M. E.:, Longitudinal variation of tides in the MLT region: 2. Relative effects of solar radiative and latent heating, J. Geophys. Res., 115, A06317, doi:10.1029/2009JA014898, 2010. 